\section{RNEE}

Revista Nicolaita de Estudios Económicos Nueva Época

Instituto de Investigaciones Económicas y Empresariales
Universidad Michoacana de San Nicolás de Hidalgo

Volumen XV, Número 2, Julio - Diciembre 2020, pp. 59-99

ISSN (Print): 1870 - 5464 ISSN (On-line): 2007 - 9877

DOI: $10.33110 /$ rnee.v15i2.316

(Recibido: 01/julio/2020; Aceptado: 27/noviembre/2020)

\title{
Actividad económica y colapso bancario: un modelo markoviano de cambio de régimen de las reservas bancarias
}

\section{Economic activity and banking collapse: Markov regime switching model of bank capital reserves}

\author{
Brenda Jovana Aguilar Hernández ${ }^{a}$ \\ Isela Elizabeth Téllez-León ${ }^{\mathrm{b}}$ \\ Francisco Venegas Martínez ${ }^{\mathrm{c*}}$
}

\section{Resumen}

Este trabajo estima mediante simulación Monte Carlo el momento (tiempo de paro) en el que BBVA Bancomer y Banorte agotaron sus reservas de capital durante 2007-2010. Para esto se utiliza se combina un modelo de reversión a la media con un modelo markoviano de cambio de régimen; siendo los regímenes son expansión y contracción de las reservas de capital. Para ello se examina la relación entre la actividad económica a través del Indicador General de la Actividad Económica (IGAE) y las reservas bancarias de capital de enero de 2007 a diciembre de 2009 en el caso de BBVA Bancomer, y de enero de 2007 a diciembre de 2010 para Banorte. Se muestra que estos dos regímenes condicionan el comportamiento de las reservas bancarias de capital. Para los periodos de estudio, la probabilidad de contracción es aproximadamente del 0.30 mientras que la probabilidad de expansión fue 0.87. Los resultados empíricos obtenidos señalan que BBVA consume en promedio sus reservas cada nueve meses y Banorte cada 22 meses. Estos resultados son utilizados para proporcionar algunas recomendaciones en materia de política bancaria útil para los reguladores.

Palabras clave: instituciones financieras, reservas de capital, modelo markoviano de cambio de régimen, agotamiento de reservas de capital.

Clasificación JEL: C24, C02, G21, G24

\begin{abstract}
\footnotetext{
a Instituto Politécnico Nacional. E-mail: brendaaguilar877@gmail.com

b Instituto Politécnico Nacional.E.mail: tellezelizabeth_2015@yahoo.com

c Instituto Politécnico Nacional.E-mail: fvenegas1111@yahoo.com.mx

* Autor de correspondencia
}

This paper is aimed at estimating through Monte Carlo simulation the time (stopping time) in which BBVA Bancomer and Banorte deplete their capital reserves during 2007-2010. To do this, a mean reversion model is combined with a Markov regime switching model; being the regimes expansion and contraction of capital 
reserves. For doing this, the relationship between the economic activity through the General Indicator of Economic Activity (IGAE, acronym in Spanish) and the bank capital reserves from January 2007 to December 2009 in the case of BBVA Bancomer, and from January 2007 to December 2010 for Banorte is analyzed. It is shown that these two regimes condition the behavior of bank capital requirements. For the periods of study, the probability of contraction is approximately 0.30 while the probability of expansion was 0.87 . The obtained empirical results indicate that BBVA consumes its reserves on average every nine months and Banorte every 22 months. These results are used to provide some useful banking policy recommendations for regulators.

Keywords: financial institutions, reserve requirement for banks, Markov regime switching model, capital reserves depletion.

JEL Codes: C24, C02, G21, G24

\section{Introducción}

Existen dos ventajas para conocer el momento en el que se agotan las reservas bancarias. Por un lado, desde el punto de vista de los bancos, el mantener un capital de reserva mayor al porcentaje mínimo establecido tiene un costo, ya que ese recurso está inactivo, y con ello se está perdiendo el costo de oportunidad de hacer uso del mismo. Además, si éste está por debajo del 8\%, la institución bancaria incurrirá en multas, las cuales van de los 30,000 a 100,000 días de salario estipuladas en la Ley de Instituciones de Crédito. Desde el punto de vista del regulador, éste estaría interesado en saber cuándo solicitar a un banco que reponga el capital requerido y de esa manera hacer más eficiente su regulación. Por lo anterior, es importante desarrollar un modelado adecuado para estimar el momento en el que se agotarán las reservas bancarias o bien para estimar la probabilidad de que eso suceda en una fecha futura.

Lo ideal para un banco sería mantener la menor cantidad posible de capital de reserva sin caer por debajo del 8\%. Para ello es fundamental tener una medición dinámica de cómo los bancos van agotando su capital de reserva, lo cual es posible mediante un modelo de un sistema de ecuaciones diferenciales estocásticas. Así, se tendrá conocimiento acerca de la tasa a la que el banco tiene que reponer su capital, de tal manera que no exista capital en exceso y se considere el efecto que la actividad económica tiene sobre las reservas bancarias de capital.

La razón por la que se analizan, particularmente, las Instituciones de Banca Múltiple Banco Bilbao Vizcaya Argentaria (BBVA) Bancomer (en adelante BBVA) y Banco Mercantil del Norte (BANORTE) es indagar si existen diferencias entre estos dos bancos en el tiempo que consumen sus reservas en un periodo d tiempo dado. Uno de ellos es extranjero y el otro nacional, lo cual implica diferente sensibilidad a la información económica que incorporan en sus modelos. Para BANORTE las decisiones son tomadas en México, mientras que para BBVA las decisiones son tomadas, esencialmente, en España. Esto tiene un particular interés en el análisis en la administración de riesgos, lo cual repercute en la estabilidad bancaria.

Las reservas bancarias de capital se toman en esta investigación como las quitas y castigos. Se le denomina quitas a los créditos otorgados por las Instituciones de Banca Múltiple que ya no tiene posibilidades de cobro y a través de las cuales se recupera un monto total o parcial del valor de la cartera crediticia. El objetivo principal de este trabajo es identificar si las reservas bancarias de capital de BBVA y BANORTE están influenciadas por el estado en que se encuentre la actividad económica, medida a través del Índice Global de Actividad Económica (IGAE). El segundo objetivo es estimar el momento (tiempo de paro) en el que BBVA y 
BANORTE agotan sus reservas bancarias de capital con la finalidad de tener grados de holgura en el requerimiento mínimo establecido por el órgano regulador ${ }^{1}$. En consecuencia se establece la siguiente hipótesis: si los dos estados (expansión y contracción) de las reservas bancarias de capital son influenciados por la actividad económica, entonces la matriz de transición entre los dos estados está influenciada por el IGAE. La hipótesis secundaria es: si las reservas bancarias de capital se encuentran en el estado de contracción, entonces el tiempo de paro estará determinado cuando las reservas están por debajo del nivel mínimo establecido por el marco regulatorio.

Esta investigación está organizada en tres apartados. En la primera sección se presenta el contexto en el que se desenvuelven las instituciones bancarias, la regulación actual y los problemas que pueden enfrentar. La segunda sección un modelo varsoviano de cambio de régimen (MS por sus siglas en inglés, Markov Switching). Se utiliza para ello el paquete Eviews8.0. Además se plantea un sistema de ecuaciones diferenciales estocásticas (EDE) para modelar el cambio de régimen. Por último, la tercera sección realiza la calibración de los parámetros de las EDE a través de modelos econométricos y simulación Monte Carlo mediante Visual Basic. Por último, se presentan las conclusiones con base en los resultados obtenidos, los alcances y las limitaciones.

\section{Administración del riesgo crédito}

En esta sección se abordarán los fundamentos de la administración del riesgo de crédito con la finalidad de dar un contexto a la forma en que se desenvuelven las instituciones bancarias en México. Se revisan las recomendaciones del Comité de Supervisión Bancaria de Basilea y los problemas que enfrentan las instituciones bancarias al tener información asimétrica. Para una institución bancaria el mayor riesgo al que se expone, dada la naturaleza de su actividad, es el riesgo de crédito. El riesgo de crédito se define como la pérdida potencial (esperada y no esperada) por e incumplimiento de la contraparte en sus obligaciones contractuales en tiempo y forma de acuerdo a los términos establecidos, lo cual también puede conducir a pérdidas cuando los deudores ven reducida su calificación por las agencias crediticias, generando así una disminución en el valor de mercado de sus obligaciones sin que necesariamente ocurra un impago. De esta manera se puede decir que el riesgo de crédito tiene dos enfoques: el primero se refiere a los activos crediticios, los cuales están expuestos al riesgo de incumplimiento y el segundo radica en los activos financieros expuestos al riesgo de contraparte. Así, se presentan dos tipos de pérdidas: las pérdidas esperadas y las pérdidas no esperadas. La pérdida esperada representa la proporción del crédito inicial que se supone se puede perder. Para propósitos de cálculo de las reservas para riesgos crediticios, la pérdida esperada se determina como el producto de la probabilidad de incumplimiento por la severidad de la pérdida en caso de incumplimiento y la exposición al incumplimiento. En tanto que, la pérdidas esperadas totales, se define como la adición de las cantidades de las pérdidas esperadas para cada una de las posiciones individuales condicionadas al riesgo de crédito (Banxico, 2010). Las pérdidas no esperadas son pérdidas inusuales en la cartera de crédito que no se consideran como posibles y que son propensas a ocurrir en el futuro teniendo consecuencias en el capital. El nivel de cobertura de esta pérdida por el capital y reservas de una Institución es un indicador de solvencia ajustada por riesgo de la misma.

\footnotetext{
${ }^{1}$ El tiempo de paro es una variable aleatoria que registra el momento en que ha ocurrido cierto evento de un proceso estocástico en el tiempo $t$.
} 
La administración del riesgo de crédito involucra todas aquellas actividades que la institución bancaria considera pertinentes y consisten en medir, minimizar y prevenir las pérdidas esperadas y las pérdidas no esperadas. Las diferentes metodologías que han sido utilizadas para medir el riesgo de crédito incluyen modelos tradicionales, estructurales y reducidos. El modelo tradicional considera el criterio subjetivo del analista formado a partir de la experiencia adquirida al asignar créditos. Se identifican dos corrientes: una utiliza una ponderación de factores y la otra emplea conceptos de tipo fundamental. Dentro de este tipo de modelos se encuentra el modelo de las 5cs (carácter, capital; capacidad, colateral y ciclo). Una desventaja es que utiliza el criterio del analista, por lo que es subjetivo y tomar una decisión se vuelve un asunto complicado. Esto es costoso para las empresas, ya que requiere de un equipo numeroso de analistas financieros. Dadas estas condiciones en contra, el modelo se ha sustituido por técnicas estadísticas y probabilísticas más sofisticadas.

Por otro lado, los modelos estructurales son más comunes en su uso. Algunos ejemplos de ellos son: modelo de Merton (1974), Geske (1977), CreditMetrics de JP Morgan and Company (1977) y CreditPortafolioManager de KMV Moody's (Crouhy, Galai, y Mark, 2000). Estos modelos suponen que se dispone de toda la información del mercado y de las empresas y que se tiene conocimiento de las deudas y del valor de los activos, pero la información bursátil algunas veces no está disponible para aquellas empresas que tienen un índice de baja, mínima o ninguna bursatilidad (Claramunt, Casanovas, y Caicedo, 2012).

Mientras que los modelos reducidos son los propuestos por: Altman (1968), logit, probit y lineales de probabilidad (Greene, 2002); modelos de simulación (Dunkel y Weber, 2007; y Ramaswany, 2005), redes neuronales artificiales (Atiya, 2001). Dichos modelos suponen que se tiene información a priori de las empresas o de los créditos clasificados como cumplidos o incumplidos en torno a las obligaciones que implica el crédito. Dado que existe asimetría en la información, esto apunta a resolver un problema de selección adversa (Akerlof, 1970). El problema surge cuando el banco en el momento de otorgar un contrato de préstamo, no tiene la capacidad de distinguir entre los riesgos en que puede incurrir con cada prestatario.

Los modelos anteriores han sido los más utilizados por las instituciones bancarias en las últimas décadas, pero en los últimos años las Instituciones de Crédito han desarrollado metodologías alternativas para la gestión de riesgos, esto a consecuencia de las modificaciones en los requerimientos de capitalización que han establecidos las autoridades regulatorias. El desarrollo de metodologías para cuantificar el riesgo de crédito ha sido menor en comparación con el riesgo de mercado. En parte, por las características propias de este tipo de riesgo. A pesar de estas limitaciones, se han creado muy diversas metodologías para estimar la probabilidad de incumplimiento (Venegas-Martínez, 2008; Cruz-Aké, Venegas-Martínez y Ortiz-Ramírez, 2010).

Las Instituciones bancarias tienen que clasificar sus operaciones sujetas a riesgo de crédito conforme al emisor o contraparte de la operación o, en su caso, al tipo de crédito de que se trate con el objetivo de alcanzar su requerimiento de capital por riesgo de crédito de acuerdo al método estándar. Asimismo deben tener conocimiento de las calificaciones otorgadas por las Instituciones calificadoras y los grados de riesgo. La Comisión Nacional Bancaria y de Valores (CNBV) es la encargada de informar sobre las actualizaciones que se llegarán hacer de las calificaciones.

La regulación en el sistema financiero mexicano ha pasado por muchos cambios. Hace tres décadas, las actividades de los bancos estaban muy restringidas por el regulador. Unos años más tarde, estas regulaciones se fueron desvaneciendo y ya no eran tan restrictivas, con lo que surgió el concepto de "Banca Universal". Unos años después empezó un periodo de privatización y se abrieron nuevos bancos y con el tratado de libre comercio de Norte América se permitió la entrada de bancos extranjeros. En seguida vino una devaluación provocando serios problemas en las variables macroeconómicas fundamentales, siendo afectadas la inflación, las tasas de interés y los salarios reales, por lo que el sistema bancario mexicano estuvo expuesto al riesgo de crédito, pero este 
riego no se administraba adecuadamente y en consecuencia el gobierno tuvo que intervenir. Después de estos acontecimientos, México tiene especial cuidado en las reglas de regulación bancaria para administrar el riesgo crédito.

\subsection{Comité de Basilea y manejo de reservas de capital}

El Comité de Supervisión Bancaria de Basilea es una organización internacional ${ }^{2}$ cuyo objetivo es salvaguardar la integridad del sistema financiero estableciendo una colección de propuestas de regulación internacionales de capital y liquidez con el objetivo de fomentar la solidez y estabilidad del sistema bancario, y así promover un crecimiento económico sostenible en un entorno de estabilidad. "El fin de estas reformas es mejorar la capacidad del sector bancario para absorber perturbaciones procedentes de tensiones financieras o económicas de cualquier tipo, minimizando con ello el riesgo de contagio desde el sector financiero hacia la economía real" (Basel Committee on Banking Supervision, 2010). Por ello, las entidades financieras están sometidas a mayores controles prudenciales y de vigilancia. La exigencia de que los bancos posean un nivel de capital, acorde con sus riesgos, es una regla prudencial fundamental, pues el capital constituye la última línea de defensa ante pérdidas inesperadas de las entidades (Elizalde y Repullo, 2004). No obstante, se debe cuidar que la normativa de suficiencia del capital no sea un motivo de disparidad competitiva con los bancos internacionales.

Las crisis han mostrado evidencia de lo importante que es una sana y fuerte supervisión bancaria con el fin de respaldar el correcto manejo de las políticas prudenciales y evitar el riesgo moral que podrían tener sobre todo las grandes instituciones financieras, además de incentivar prácticas severas de gestión del riesgo (Caruana, 2010). Después de la crisis se hicieron evidentes una serie de deficiencias que deben resolver las partes involucradas en el sector crediticio, fundamentalmente, las instituciones que participan en el mercado de crédito y que enfrentan el reto de administrar la asignación de las reservas de capital.

La administración adecuada de las reservas de capital ha sido un tema central para el Comité de Supervisión Bancaria desde 1988 cuando se emitió el Acuerdo de Capital de Basilea y su objetivo era determinar el cálculo de la reserva de capital por riesgo de crédito para los sistemas bancarios. Las instituciones bancarias han aprobado los tres pilares en los que se basa el marco de Basilea II, los cuales han sido reforzados en Basilea III, de diciembre del 2010, que resumen los siguientes temas: 1) cálculo de los requerimientos mínimos de capital; 2) proceso de examen de supervisor; y 3) disciplina del mercado.

Los componentes del capital regulado, con los ajustes regulatorios asociados según Basilea III, están conformados por los siguientes elementos sujetos a sus correspondientes límites, los cuales entraron en vigor el 1 de enero de 2015: capital ordinario de nivel I y deberá ascender al menos al 4.5\% de los activos ponderados por riesgo en todo momento; capital adicional de nivel II y deberá ascender al menos al 6.0\% de los activos ponderados por riesgo en todo momento; capital total (capital del nivel I más capital del nivel II) deberá ascender al menos al 8.0\% de los activos ponderados por riesgo en todo momento. Adicionalmente, los bancos deberán mantener "conservación de capital" del 2.5\% que estará compuesto de capital ordinario, se añadirá al

\footnotetext{
${ }^{2}$ El Comité de Supervisión Bancaria de Basilea creado en 1975 está conformado por los principales representantes de autoridades de supervisión bancaria y de bancos centrales de varios países. Su Secretaría está ubicada en Basilea (Suiza) y sus reuniones se llevan a cabo en la sede del Banco de Pagos.
} 
requerimiento mínimo del 4.5\%, y se aplicará paulatinamente a partir del 1 de enero de 2016 y entrará completamente en vigor el 1 de enero de 2019. De esta manera, con base en el Acuerdo de Basilea III, los bancos asumiendo el nuevo requerimiento de capital ordinario deberán mantener en situaciones normales al menos un $7 \%$ de capital ordinario, incluyendo un colchón de conservación de capital del 2.5\%. El requerimiento de capital total se mantiene en el 8\% actual, por lo que no necesita ningún periodo de adaptación.

\subsection{Problemas de agencia en la regulación de capital}

La teoría de la agencia posibilita estudiar la problemática entre personas, entorno y recursos. En un contrato de agencia siempre existe un contrato en el que una persona (principal) le pide a otra (agente) se encargue de la administración de actividades y la toma de decisiones dejándola en la libertad total para ejercerlas y velando por los intereses del principal, pero este último no conoce al detalle las actividades que el agente realiza, solo conoce los resultados finales. En relación al nivel de riesgos, ambos agentes están expuestos a este problema, pero quien asume el mayor nivel de riesgo es el principal, ya que delega la toma de decisiones al agente. Esto se puede resolver al conciliar ambos intereses y obtener mayor información sobre las tareas y conducta del agente.

\section{Calibración de la ecuación diferencial estocástica de las reservas}

Una vez descrito el entorno en el que se encuentran las instituciones bancarias, las obligaciones que tiene que cumplir y los problemas a los que se enfrentan, el siguiente paso es presentar algunos conceptos relacionados con la metodología de Markov Switching (MS), volatilidad estocástica (SV) y sistemas de ecuaciones diferenciales estocásticas (EDE) mismos que serán utilizados a continuación en el análisis de la dinámica de cada una de las variables.

\subsection{Modelos de cambios de régimen (MS)}

El comportamiento de la mayoría de las series económicas puede mostrar cambios abruptos durante el transcurso del tiempo debido a diversos factores, tales como una crisis financiera o una política gubernamental, entre otras. Un cambio inesperado en el comportamiento histórico de la variable $X_{t}$ se puede expresar matemáticamente como un vector autorregresivo de primer orden de la siguiente manera: $X_{t}=a_{1}+\phi X_{t-1}+\varepsilon_{t}$ con $\varepsilon_{t} \sim N\left(0, \sigma^{2}\right)$ y $t=0,1, \ldots, t_{0}$. Después del tiempo $t_{0}$ ocurre nuevamente un cambio importante y se puede describir mediante: $X_{t}=a_{2}+\phi X_{t-1}+\varepsilon_{t}$ para $t_{0}=t_{0}+1, t_{0}+2 \ldots$ Es importante destacar que hay una forma de expresar ambos comportamientos en un modelo generalizado $X_{t}=a_{s_{t}}+\phi X_{t-1}+\varepsilon_{t}$ donde $s_{t}$ es una variable aleatoria (variable de estado) con $s_{t}=1$ para $t=0,1, \ldots, t_{0}$ y $s_{t}=2$ para $t_{0}=t_{0}+1, t_{0}+2 \ldots$ con residuos normalmente distribuidos con media cero y la varianza dependiente del estado en el que se encuentre, es decir, $\varepsilon_{t} \sim N\left(0, \sigma_{s_{t}}^{2}\right)$. Se define a $s_{t}$ como el régimen en el que se encuentra un proceso en la fecha $t$, pueden existir $N$ régimenes posibles pero en este caso $s_{t}=1,2$, en el periodo de estudio y es una realización de una cadena de Markov de primer orden con dos estados con probabilidades de transición:

$$
\mathrm{P}\left(s t=\left.j\right|_{s t_{-} 1}=i, s t_{-} 2=k, \ldots s t_{-}, s t_{-2}, \ldots\right)=\mathrm{P}\left(s t=\left.j\right|_{s t_{-} 1}=i\right)=p_{i j}, i, j=1,2, \ldots
$$


Se supone que la probabilidad de un cambio de régimen depende sólo del pasado a través del valor de régimen más reciente. Es decir, la probabilidad de transición, $p_{i j}$, de pasar del estado $s_{t-1}=i$ al estado $s_{t}=j$ depende solo del estado en el tiempo inmediato anterior, $t-1$, y no de estados más lejanos en el estado, $t-2$, $t-3, \ldots$ Estas probabilidades de transición satisfacen la condición $p_{i 1}+p_{i 2}=1$ y se escriben en un arreglo matricial de la siguiente manera:

$$
\begin{array}{lll}
P & p_{11} & p_{12} \\
& p_{21} & p_{22}
\end{array}
$$

La matriz Pes llamada la matriz de probabilidades de transición de la cadena de Markov. La inferencia acerca del valor de la variable de estado $s_{t}$ se basa en la historia de $X_{t}$ y el conocimiento de los parámetros $\theta$. Estas probabilidades, en forma vectorial para los estados posibles, están dadas por:

$$
\xi_{j t}=\left[\begin{array}{l}
\mathrm{P}\left(s_{t}=1 \mid x_{t}, x_{t-1}, \ldots, x_{1}, x_{0} ; \theta\right) \\
\mathrm{P}\left(s_{t}=2 \mid x_{t}, x_{t-1}, \ldots, x_{1}, x_{0} ; \theta\right)
\end{array}\right]
$$

Esta inferencia es realizada iterativamente para el conjunto de observaciones obtenidas en los tiempos $t=1,2, \ldots, T$ Las magnitudes que se necesitan para hacer estas iteraciones son las densidades de $x_{t}$ condicional a cada régimen posible:

$$
\eta_{j t}=f\left(x_{t} \mid s_{t}=j ; x_{t-1}, \ldots, x_{1}, x_{0} ; \theta\right)=\frac{1}{\sqrt{2 \pi \sigma^{2}}} \exp \left[-\frac{\left(x_{t}-a_{j}-\phi x_{t-1}\right)^{2}}{2 \sigma^{2}}\right]
$$

Específicamente, dado el resultado de la expresión:

$$
\xi_{i, t-1}=\left[\begin{array}{l}
\mathrm{P}\left(s_{t-1}=1 \mid x_{t-1}, \ldots, x_{1}, x_{0} ; \theta\right) \\
\mathrm{P}\left(s_{t-1}=2 \mid x_{t-1}, \ldots, x_{1}, x_{0} ; \theta\right)
\end{array}\right]
$$

Se puede calcular la densidad condicional de la t-ésima observación mediante:

$$
f\left(x_{t} \mid x_{t-1}, \ldots, x_{1}, x_{0} ; \theta\right)=\sum_{i=1}^{2} \sum_{j=1}^{2} p_{i j} \xi_{i, t-1} \eta_{j t}
$$

entonces

$$
\xi_{j t}=\frac{\sum_{i=1}^{2} p_{i j} \xi_{i, t-1} \eta_{j t}}{f\left(x_{t} \mid x_{t-1}, \ldots, x_{1}, x_{0} ; \theta\right)}
$$

Como resultado de la ejecución de estas interacciones, se puede evaluar el logaritmo de la función de verosimilitud con respecto a los parámetros y se define como:

$$
\ln f\left(x_{1}, x_{2}, \ldots, x_{T} \mid x_{0}, \theta\right)=\sum_{t=1}^{T} \ln f\left(x_{t} \mid x_{t-1}, \ldots, x_{1}, x_{0} ; \theta\right)
$$


Así, una estimación para el valor de los parámetros se puede realizar maximizando la anterior función por medio de métodos numéricos si es que no existe una solución analítica. Lo que interesa es hacer inferencia sobre los distintos regímenes que presenta la serie $X_{t}$ a partir de un conjunto de observaciones a través del tiempo por lo que se desea obtener $P\left(s_{t}=j \mid X_{t} ; \theta\right)$ con $j=1,2$ y $t=1,2, \ldots$, . Aplicando el teorema de Bayes:

$$
P\left(s_{t}=j \mid X_{t} ; \theta\right)=\frac{P\left(s_{t}=j ; \theta\right) f\left(X_{t} \mid s_{t}=j ; \theta\right)}{f\left(X_{t} ; \theta\right)}=\frac{P\left(s_{t}=j ; \theta\right) f\left(X_{t} \mid s_{t}=j ; \theta\right)}{\sum_{j=1}^{2} f\left(X_{t} \mid s_{t}=j\right) P\left(s_{t}=j\right)}
$$

\subsection{Modelos de volatilidad estocástica}

La dispersión de los datos durante el periodo de análisis puede ser mayor en comparación con otros periodos. Por tal motivo, es importante determinar un patrón de comportamiento estadístico de la volatilidad a lo largo del horizonte de estudio. Entre los modelos no lineales se encuentran los modelos ARCH y GARCH. Un proceso $\mathrm{ARCH}(\mathrm{p})$ tiene la siguiente expresión:

$$
\sigma_{t}^{2}=\alpha_{o}+\alpha_{1} \varepsilon_{t-1}^{2}+\alpha_{2} \varepsilon_{t-2}^{2}+\cdots+\alpha_{p} \varepsilon_{t-p}^{2}, \sigma_{t}^{2}=\alpha_{o}+\sum_{i=1}^{p} \alpha_{i} \varepsilon_{t-i}^{2}
$$

donde $\alpha_{o}>0$ es la mínima varianza condicional a ser observada $\alpha_{i} \geq 0$ es una condición suficiente y necesaria para la existencia de la varianza condicional e incondicional, $\varepsilon_{t}=\sigma_{t} a_{t}$ con $\sigma_{t}$ y $a_{t}$ indipendientes y $a_{t} \sim N(0,1)$. Un proceso GARCH $(p, q)$ satisface:

$$
\begin{aligned}
& \sigma_{t}^{2}=\alpha_{o}+\alpha_{1} \varepsilon_{t-1}^{2}+\alpha_{2} \varepsilon_{t-2}^{2}+\cdots+\alpha_{p} \varepsilon_{t-p}^{2}+\theta_{1} \varepsilon_{t-1}^{2}+\theta_{2} \varepsilon_{t-2}^{2}+\cdots+\theta_{q} \varepsilon_{t-q}^{2} \\
& \sigma_{t}^{2}=\alpha_{o}+\sum_{i=1}^{p} \alpha_{i} \varepsilon_{t-i}^{2}+\sum_{j=1}^{q} \theta_{j} \varepsilon_{t-j}^{2},
\end{aligned}
$$

donde $\alpha_{o}>0, \alpha_{i} \geq 0, \theta_{j} \geq 0$ con $i=1,2,3, \ldots, p$ y $j=1,2,3, \ldots, q ; \sum_{i=1}^{p} \alpha_{i}+\sum_{j=1}^{p} \theta_{j}<1$, $\varepsilon_{t}=\sigma_{t} a_{t}$ con $\sigma_{t}$ y $a_{t}$ independientes y $a_{t} \sim N(0,1)$. Por otro lado, para el desarrollo de modelos con varianza condicional asimétrica se recurre a los modelos EGARCH y TGARCH, por mencionar algunos. El modelo univariante TGARCH se define

$$
\sigma_{t}^{2}=\alpha_{o}+\alpha_{1} \varepsilon_{t-1}^{2}+\gamma d_{t-1} \varepsilon_{t-2}^{2}+\theta_{1} \sigma_{t-1}^{2}, \text { donde } d_{t-1}= \begin{cases}0 & \text { si } \varepsilon_{t-1} \geq 0 \\ 1 & \text { si } \varepsilon_{t-1}<0\end{cases}
$$

En las últimas décadas se han desarrollado, estudiado y aplicado los modelos de volatilidad estocástica con el objetivo de modelar la volatilidad que está relacionada a los datos de las series de tiempo financieras. La serie $X_{t}$ con $t=1,2, \ldots, N$ se puede modelar, siguiendo a Taylor (1982), como $X_{t}=\sigma_{t} \varepsilon_{t}$. Con un proceso $\operatorname{AR}(1)$ de primer orden expresado como $h_{t+1}=\mu_{0}+\mu_{1} h_{t}+\eta_{t}$, el modelo univariado satisface:

$$
X_{t}=e^{1 / 2 h} \varepsilon_{t}
$$




\subsection{Sistemas de ecuaciones diferenciales estocásticas}

Un proceso de difusión con reversión a la media se representa por medio de la ecuación diferencial estocástica:

$$
\mathrm{d} K_{t}=a\left(b-K_{t}\right) \mathrm{d} t+\sigma_{K} \mathrm{~d} W_{t}
$$

donde $K_{t}$ es la reserva de capital, $W_{t}$ es un movimiento browniano ${ }^{3}$. El parámetro $b$ es la media y el parámetro a es la velocidad de ajuste hacia la media.

\section{Simulación Monte Carlo de los tiempos de paro}

La presente investigación utiliza tres series de tiempo, las quitas y castigos tanto de BBVA Bancomer como de BANORTE y el IGAE. Se plantean dos modelos: en el primero, MS1, se establece como variable explicativa el IGAE y variable explicada las quitas y castigos de BBVA Bancomer, y en el segundo, MS2, se tiene como variable explicativa el IGAE y variable explicada las quitas y castigos de BANORTE. El periodo de estudio es 2007-2010. Posteriormente, se realizan pruebas de raíces unitarias. Para ello se utiliza un análisis gráfico del correlograma y las pruebas de Dickey-Fuller aumentada (ADF) y Phillips-Perron (PP).

A continuación se estiman uno de los modelos MS, después se determinan las probabilidades de transición de cada uno de los estados, es decir, expansión y contracción. Se obtienen los parámetros para calibrar el sistema de ecuaciones diferenciales estocásticas. Por último, se realiza la simulación Monte Carlo de los tiempos de paro asociada al consumo de las reservas bancarias de capital con el fin de determinar las veces que BBVA Bancomer y Banorte descienden por debajo del límite establecido por la Ley de Instituciones de Crédito.

\subsection{Estadística descriptiva}

La estadística descriptiva sintetiza y presenta las principales características de las series bajo estudio. Se consultaron dos fuentes: la primera de ellas es el portafolio de información de la situación financiera de banca múltiple en el documento 040-1A-R19 denominado Cartera total de la CNBV. La segunda fuente fue la base de datos del Instituto Nacional de Estadística y Geografía (INEGI) para consultar el Indicador Global de la Actividad Económica (IGAE). El periodo de estudio es de enero de 2007 a diciembre de 2010, con un total de 48 observaciones para cada una de las variables. La información disponible, para el caso de las quitas y castigos, no permite considerar un intervalo de tiempo más amplio ${ }^{4}$. El Cuadro 1 muestra las nomenclaturas utilizadas en el resto de esta investigación.

\footnotetext{
${ }^{3}$ Véase al respecto Venegas-Martínez (2008).

${ }^{4}$ La base de datos está disponible al lector.
} 
Notación de las variables empleadas en los modelos

\begin{tabular}{ll}
\hline Nomenclatura & \multicolumn{1}{c}{ Concepto } \\
\hline Bbva & Quitas y castigos de Bbva \\
Banorte & Quitas y castigos de Banorte \\
IGAE & Indicador Global de la Actividad Económica \\
\hline
\end{tabular}

Fuente: Elaboración propia

El Cuadro 2 muestra las variables utilizadas en cada uno de los modelos. Para la construcción de las variables dependientes se emplearon la base de datos denominadas quitas y castigos de BBVA para el primer modelo MS1, así como quitas y castigos de Banorte para el segundo modelo MS2. En el caso de la variable independiente IGAE se consultó la serie histórica en el sitio web del INEGI ${ }^{5}$. El Cuadro 3 define cada una de las variables.

Cuadro 2

Variables empleadas en los modelos MS1 y MS2

\begin{tabular}{cccc}
\hline \multicolumn{2}{c}{ Modelo MS1 } & \multicolumn{2}{c}{ Modelo MS2 } \\
\hline Variable Independiente & r_IGAE & Variable Independiente & r_IGAE \\
Variable Dependiente & r_Bbva & Variable Dependiente & r_Banorte \\
\hline
\end{tabular}

Fuente: Elaboración propia

Cuadro 3

Descripción de las variables usadas en los modelos MS

\begin{tabular}{cl}
\hline Variable & Definición \\
\hline r_Bbva & $\begin{array}{l}\text { Una quita es el porcentaje de deuda que condona voluntariamente el acreedor al deudor. } \\
\text { Un castigo se da cuando el acreedor, no por voluntad a diferencia de la quita, decide } \\
\text { anular el crédito ya que se ha determinado que no hay posibilidades de recuperación } \\
\text { del crédito, aunque se buscará recuperar el crédito castigado }\end{array}$ \\
r_IGAE & Es un Indicador mensual de la dinámica del sector real en el corto plazo \\
\hline
\end{tabular}

Fuente: Elaboración propia

Quitas y castigos están expresados en millones de pesos. La serie de tiempo IGAE es una serie desestacionalizada con índice base 2008. 
Las variables que se utilizaron en la estimación de los dos modelos MS1 y MS2 fueron analizadas de manera individual. Este estudio consistió en el análisis gráfico como el correlograma y análisis estadístico mediante las pruebas de raíz unitaria ADF Y PP (ver anexos 1, 2 y 3). Se observa la presencia de raíz unitaria en las tres series analizadas, por ello se realizan transformaciones a las series para eliminar tendencia y volatilidad en cada una de ellas para obtener resultados robustos ya que es recomendable trabajar con variables estacionarias. Las series en niveles presentan raíz unitaria, es decir, las series de tiempo son no estacionarias, pero una vez realizadas las transformaciones se tuvieron series estacionarias y la interpretación de dicha transformación a las series se interpreta como rendimientos de cada una de las variables bancarias y como tasa de crecimiento del IGAE. De esta manera, se aplicaron las transformaciones correspondientes en las variables.

El Cuadro 4 muestra la estadística descriptiva de las variables. En el caso de la media, se puede observar que r_IGAE es 0.00109 y que hay una diferencia de 0.06537 entre la media de r_Bbva y de r_Banorte, lo cual se podría interpretar como un promedio similar. La desviación estándar de r_IGAE está cerca del cero, es decir, sus valores son semejantes, por lo que no hay gran variación entre sus valores pero para r_Bbva y r_Banorte, existe una dispersión de 0.42864 y de 0.55883 , respectivamente. En el mes de junio del 2009 se registró el valor máximo para r_IGAE. En el caso de r_Bbva, aconteció en diciembre del 2010, y para r_Banorte se registró en abril del 2008. El valor mínimo de r_IGAE fue en el mes de enero del 2009, para r_Bbva en el mes de enero del 2008, y para r_Banorte sucedió en junio del 2009

Cuadro 4

\begin{tabular}{ccccc}
\hline Variable & Media & Desv. estándar & Máximo & Mínimo \\
\hline r_IGAE & 0.00109 & 0.00773 & 0.001653 & -0.02240 \\
r_Bbva & 0.11218 & 0.42864 & 1.97504 & -0.66749 \\
r_Banorte & 0.17754 & 0.55883 & 1.62141 & -0.81175 \\
\hline
\end{tabular}

Fuente: Elaboración propia con datos de CNBV e INEGI

Para r_IGAE sólo le llevó seis meses pasar de su valor mínimo al máximo, pero no pasó lo mismo con r_Bbva, fueron 23 meses los que transcurrieron, y en el caso de r_Banorte pasó de su valor máximo al mínimo en 14 meses. Con lo que se puede deducir que estás dos últimas variables presentan un comportamiento diferente, posiblemente por la administración de riesgos desigual que tienen dichas instituciones financieras.

Otro elemento de la estadística descriptiva es la prueba denominada Jarque-Bera (JB) El objetivo de esta prueba es comparar la relación entre los coeficientes de asimetría y curtosis de los residuos con los de una distribución normal ${ }^{6}$. Las hipótesis a contrastar son $H_{0}$ : los residuos están normalmente distribuidos y $H_{1}$ : los residuos no están normalmente distribuidos. Se rechaza la hipótesis nula si el valor de $p$, nivel de probabilidad, es cercano a cero, en otras palabras, si $p<0.05$ no se acepta $H_{0}$ con un nivel de confianza del $95 \%$ y si $p<0.10$ se rechaza $H_{0}$ con un nivel de confianza del $90 \%$. El valor del estadístico JB dista de cero en gran

\footnotetext{
${ }^{6}$ Otro elemento recurrente para detectar normalidad en los residuos son los histogramas de las series de tiempo.
} 
medida, por lo que se puede afirmar que los residuos no se distribuyen normalmente. En el Cuadro 5 se presentan los resultados de la prueba JB para cada una de las variables. Se puede observar que el valor del estadístico JB dista del cero más de cuatro unidades para r_IGAE y r_Banorte, mientras que para $\mathrm{r}_{-}$Bbva la diferencia es mucho mayor. Además, la probabilidad es menor a 0.10 para r_IGAE y r_Banorte, con lo que se concluye que los residuos de cada variable se distribuye normalmente con un nivel de confianza del 90\%, excepto para Bbva donde el nivel de confianza es $95 \%^{7}$.

Cuadro 5

\begin{tabular}{lll}
\hline Variable & Jarque-Bera & Probabilidad \\
\hline r_IGAE & 4.78764 & 0.09128 \\
r_Bbva & 116.77030 & 0.00000 \\
r_Banorte & 5.37807 & 0.06795 \\
\hline
\end{tabular}

Fuente: Elaboración propia con datos de CNBV e INEGI

Como se mencionó anteriormente, el comportamiento histórico de los datos se puede expresar como un proceso autorregresivo $\mathrm{AR}(p)$ (Hamilton, 2005). Es por ello, que en esta sección se presentan los resultados de las pruebas econométricas que permiten deducir el proceso $\operatorname{AR}(p)$, el cual será utilizado para la estimación de los modelos MS1 y MS2 siguiendo la metodología Box-Jenkins. El primer paso es el análisis gráfico, posteriormente la ratificación de la no existencia de raíz unitaria, utilizando las herramientas como el correlograma y las pruebas $\mathrm{ADF}$ y PP y por último, la estimación del proceso $\mathrm{AR}(p)$. En seguida se describe la evolución de las series de tiempo consideradas en la estimación.

La Gráfica 1 muestra la evolución de r_IGAE mensual (la tasa de crecimiento del IGAE) durante el periodo 2007-2010, con un total de 47 observaciones. Se puede observar que hay un intercepto con valor positivo, no tiene tendencia ni volatilidad. La serie analizada luce como una serie estacionaria ya que existe una variación mínima alrededor de su media. De febrero a octubre del 2007, es decir, los primeros nueve meses, se registraron rendimientos positivos, por otro lado, de los 47 datos, sólo 15 fueron negativos, es decir, el 32\% del total, con un promedio de -0.00720. También, se observa un valor mínimo de - 0.02240 que se dio en enero del 2009, mientras que su valor máximo, 0.01653, fue en junio del 2009. Los valores extremos ocurrieron el mismo año y le llevó seis meses recuperarse. Esta caída puede ser consecuencia de la crisis 2007-2008.

\footnotetext{
${ }^{7}$ Las Gráficas de la densidad Kernel confirman la no normalidad en las variables (ver anexo 6).
} 


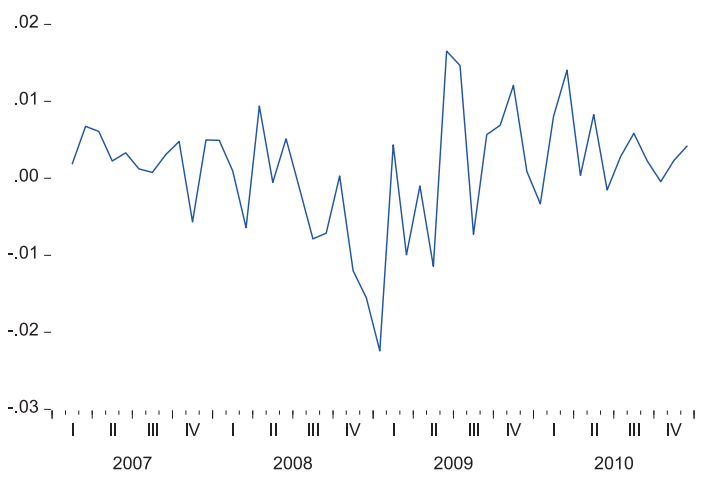

Fuente: Elaboración propia con datos de CNBV e INEGI

La Gráfica 2 muestra la evolución de r_Bbva durante el periodo 2007-2010. Se observa que tiene intercepto negativo y no presenta tendencia, ni volatilidad, justo las características que tiene una serie de tiempo estacionaria. Se observan las fechas donde acontecieron los valores mínimo y máximo antes mencionados en la estadística descriptiva, los cuales pasaron en enero de 2008 y diciembre de 2010, respectivamente. Durante este periodo acontecieron dos eventos en los que descendieron y aumentaron los valores considerablemente en tan sólo un mes. El primero de ellos fue en diciembre del 2012, a una distancia de 0.93193 del valor máximo, para descender el siguiente mes a 0.28085 su valor mínimo y el segundo aconteció en diciembre del 2009 que dista de su valor máximo 1.37348 y descendió al siguiente mes a 0.24253 de su valor mínimo. Estos tres acontecimientos tuvieron lugar al final e inicio de cada año. También, se puede ver que existen valores negativos, lo que posiblemente refleja que Bancomer no contaba, en ese momento, con capital para hacer frente a sus obligaciones. Por último, de noviembre a diciembre de 2010 se da un cambio con un valor de 1.94290, siendo éste el cambio más grande que se registró.

Gráfica 2

Evolución del r_Bbva 2007 - 2010

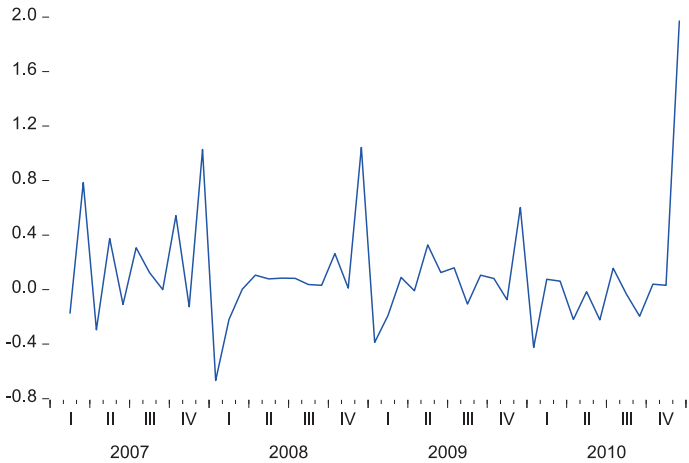

Fuente: Elaboración propia con datos de CNBV e INEGI 
La Gráfica 3 muestra la evolución de r_Banorte durante el periodo comprendido entre 2007 al 2010. Se advierte que tiene intercepto negativo, carece de tendencia y volatilidad, se verá que la serie de tiempo estacionaria, lo cual se confirmará con el análisis del correlograma y las pruebas estadísticas ADF y PP que se presentan en la siguiente sección. De igual manera, se pueden confirmar los valores mínimo y máximo que acontecieron en junio del 2009 y en abril del 2008, respectivamente. Se observa que la serie presenta datos que sobrepasan la media, la cual tiene un valor de 0.17754 . En especial, sobresalen cuatro valores con una distancia en promedio de 1.30282. Cada uno de estos valores ocurrieron en un mes, el primero ocurrió de abril a mayo del 2007, y el segundo fue de marzo a abril del 2008, con diez meses de diferencia entre estos dos primeros valores, dos meses después, sucedió el tercer valor de junio a julio del 2008, y el último, aconteció de noviembre a diciembre del 2010, es decir, pasaron quince meses sin cambios considerables.

Gráfica 3 Evolución del r_Banorte 2007 - 2010

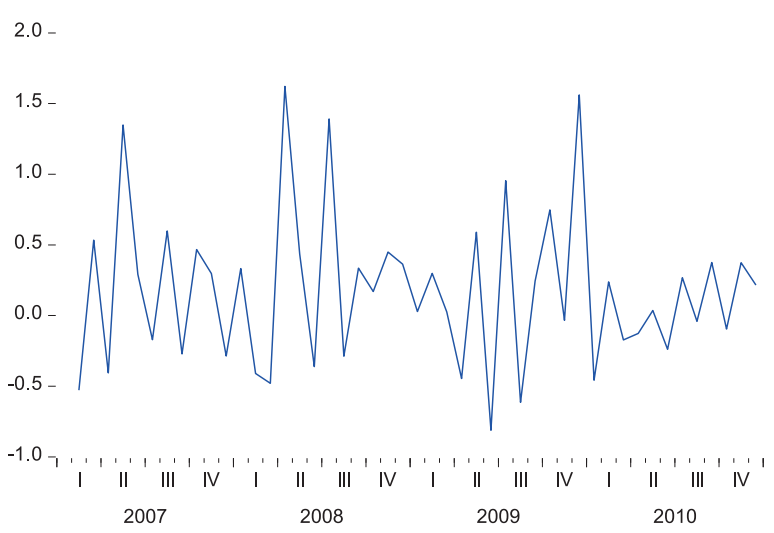

Fuente: Elaboración propia con datos de CNBV e INEGI

Otra herramienta que es útil para la identificación de la estacionariedad en una variable es el correlograma. Existen dos tipos de correlogramas: el correlograma poblacional y muestral. El primero es la gráfica de la función de autocorrelación (FAC) con respecto del rezago $k$ y el segundo es la gráfica de la función de autocorrelación muestral (FACM) con respecto del rezago $k$. La La Gráfica 4 muestra el correlograma de r_IGAE. Las barras que se observan del lado izquierdo es la autocorrelación (AC) y las segundas barras son la autocorrelación parcial (PAC). La línea continua que divide las barras representa el eje cero, las barras que se encuentran del lado derecho de este eje son valores positivos, mientras que los del lado izquierdo son valores negativos. Los primeros veinte números naturales que se muestran, después del segundo diagrama, representan los rezagos utilizados en la prueba. En seguida se observan los valores de la autocorrelación, autocorrelación parcial, el estadístico $Q$ y el valor de la probabilidad ${ }^{8}$. Una de las características más sobresalientes de los correlogramas es que muestran la estacionariedad de las series. En la Gráfica 4 se observa que los valores de PAC y AC varían alrededor del eje cero, por lo que se concluye que la serie de tiempo r_IGAE es estacionaria.

${ }^{8}$ Con el estadístico $Q$ se prueba la hipótesis conjunta de que la función de autocorrelación muestral es cero. 


\begin{tabular}{|c|c|c|c|c|c|c|}
\hline Autocorrelation & Partial Correlation & & $A C$ & $P A C$ & Q-Stat & Prob \\
\hline$\square$ & I $\square$ & 1 & 0.205 & 0.205 & 2.1109 & 0.146 \\
\hline । $\square$ । & I 口 I & 2 & 0.172 & 0.136 & 3.6334 & 0.163 \\
\hline । $\square$ । & I I I & 3 & 0.170 & 0.119 & 5.1473 & 0.161 \\
\hline 1 & $1 \square$ & 4 & 0.419 & 0.375 & 14.546 & 0.006 \\
\hline I । & $1 \mid 1$ & 5 & 0.147 & -0.001 & 15.734 & 0.008 \\
\hline । । & $\square \quad$ I & 6 & -0.142 & -0.330 & 16.859 & 0.010 \\
\hline I । & I & 7 & -0.179 & -0.324 & 18.699 & 0.009 \\
\hline 111 & $1 \mid 1$ & 8 & 0.081 & 0.007 & 19.090 & 0.014 \\
\hline l 1 1 & I I I & 9 & -0.001 & 0.104 & 19.090 & 0.024 \\
\hline । । & I & 10 & -0.135 & 0.168 & 20.226 & 0.027 \\
\hline 口 1 & 1 | 1 & 11 & -0.270 & -0.055 & 24.898 & 0.009 \\
\hline | | | & ID I & 12 & -0.081 & -0.177 & 25.325 & 0.013 \\
\hline I 1 1 & 101 & 13 & -0.008 & -0.148 & 25.329 & 0.021 \\
\hline I । & I | I & 14 & -0.104 & -0.056 & 26.080 & 0.025 \\
\hline I । & I I I & 15 & -0.212 & 0.060 & 29.315 & 0.015 \\
\hline । । & 101 & 16 & -0.233 & -0.109 & 33.354 & 0.007 \\
\hline $1 \mid 1$ & I I I & 17 & -0.014 & -0.013 & 33.368 & 0.010 \\
\hline | I | & I I I & 18 & -0.058 & -0.047 & 33.635 & 0.014 \\
\hline I I & $1 \mid 1$ & 19 & -0.185 & -0.077 & 36.436 & 0.009 \\
\hline 10 । & I I I & 20 & -0.195 & -0.023 & 39.688 & 0.005 \\
\hline
\end{tabular}

Fuente: Elaboración propia con datos de CNBV e INEGI

Los correlogramas que se muestran en las Gráficas 5 y 6 corresponden a las series de tiempo r_Bbva y r_Banorte, incluyen 47 observaciones y 20 rezagos. Para r_Bbva el valor de PAC en el primer rezago es de -0.264 (el cual no es significativo) y las barras del diagrama AC varían alrededor del eje cero y tienen forma sinusoidal, por lo que se tienen indicios de que la serie es estacionaria. Para r_Banorte el valor de PAC en el primer rezago es de -0.463 (no es significativo) y los AC varían alrededor del eje cero con forma sinusoidal, se puede concluir que la serie es estacionaria.

\section{Gráfica 5}

Correlograma de r_Bbva

\begin{tabular}{|c|c|c|c|c|c|c|c|}
\hline Autocorrelation & Partial Corr & relation & & $A C$ & $P A C$ & Q-Stat & Prob \\
\hline - 1 & m & 1 & 1 & -0.264 & -0.264 & $3.495 \ldots$ & 0.062 \\
\hline 101 & 11 & 1 & 2 & 0.110 & 0.043 & $4.116 \ldots$ & 0.128 \\
\hline 101 & 10 & 1 & 3 & -0.124 & -0.092 & $4.926 \ldots$ & 0.177 \\
\hline 11 & । & 1 & 4 & -0.069 & -0.138 & $5.184 \ldots$ & 0.269 \\
\hline 101 & 10 & 1 & 5 & 0.130 & 0.102 & $6.113 \ldots$ & 0.295 \\
\hline 1 & $1 \square$ & 1 & 6 & -0.162 & -0.116 & $7.583 \ldots$ & 0.270 \\
\hline 101 & 11 & 1 & 7 & 0.130 & 0.030 & $8.549 \ldots$ & 0.287 \\
\hline 101 & $1 \square$ & 1 & 8 & -0.195 & -0.138 & $10.80 \ldots$ & 0.213 \\
\hline 111 & 11 & 1 & 9 & 0.079 & -0.021 & $11.18 \ldots$ & 0.264 \\
\hline 101 & 10 & 1 & 10 & -0.093 & -0.092 & $11.71 \ldots$ & 0.304 \\
\hline 1 & D & 1 & 11 & -0.235 & -0.326 & $15.24 \ldots$ & 0.171 \\
\hline $1 \square$ & $1 \square$ & 1 & 12 & 0.377 & 0.267 & $24.61 \ldots$ & 0.017 \\
\hline 101 & 11 & 1 & 13 & -0.157 & 0.014 & $26.29 \ldots$ & 0.016 \\
\hline 111 & । & 1 & 14 & 0.060 & -0.180 & $26.55 \ldots$ & 0.022 \\
\hline $1 \quad 1 \quad 1$ & 10 & 1 & 15 & -0.022 & 0.097 & $26.58 \ldots$ & 0.032 \\
\hline 101 & 10 & 1 & 16 & -0.117 & -0.134 & $27.60 \ldots$ & 0.035 \\
\hline 111 & 10 & 1 & 17 & 0.081 & -0.108 & $28.11 \ldots$ & 0.044 \\
\hline 101 & 11 & 1 & 18 & -0.091 & -0.004 & $28.77 \ldots$ & 0.051 \\
\hline I & 11 & 1 & 19 & 0.172 & 0.020 & $31.19 \ldots$ & 0.038 \\
\hline 1 - 1 & 11 & 1 & 20 & -0.111 & 0.014 & $32.24 \ldots$ & 0.041 \\
\hline
\end{tabular}

Fuente: Elaboración propia con datos de CNBV e INEGI 


\begin{tabular}{|c|c|c|c|c|c|c|}
\hline Autocorrelation & Partial Correlation & & $A C$ & $P A C$ & Q-Stat & Prob \\
\hline 口 & $\square$ & 1 & -0.463 & -0.463 & 10.721 & 0.001 \\
\hline । $\mathbf{0}$ । & 111 & 2 & 0.169 & -0.057 & 12.185 & 0.002 \\
\hline 101 & $1 \square_{1}$ & 3 & 0.113 & 0.216 & 12.852 & 0.005 \\
\hline - 1 & $=1$ & 4 & -0.343 & -0.265 & 19.169 & 0.001 \\
\hline । & 111 & 5 & 0.239 & -0.071 & 22.310 & 0.000 \\
\hline । & 1 - 1 & 6 & -0.228 & -0.136 & 25.237 & 0.000 \\
\hline 1 I 1 & $1 \quad 11$ & 7 & 0.062 & -0.027 & 25.459 & 0.001 \\
\hline 1 I 1 & 101 & 8 & -0.035 & -0.141 & 25.534 & 0.001 \\
\hline 1 1 & 101 & 9 & -0.132 & -0.190 & 26.586 & 0.002 \\
\hline 1 I 1 & 101 & 10 & 0.058 & -0.202 & 26.795 & 0.003 \\
\hline $\begin{array}{lll}1 & 1 & 1\end{array}$ & 111 & 11 & 0.029 & 0.047 & 26.851 & 0.005 \\
\hline I I 1 & 1 I & 12 & 0.063 & 0.101 & 27.112 & 0.007 \\
\hline $\begin{array}{lll}1 & 1 & 1\end{array}$ & $\begin{array}{lll}1 & 1 & 1\end{array}$ & 13 & 0.008 & -0.028 & 27.116 & 0.012 \\
\hline 1 11 & 11 & 14 & 0.027 & -0.083 & 27.167 & 0.018 \\
\hline $\begin{array}{lll}1 & 1 & 1\end{array}$ & $\begin{array}{lll}1 & 1 & 1\end{array}$ & 15 & 0.001 & -0.006 & 27.167 & 0.027 \\
\hline 111 & 111 & 16 & -0.018 & 0.012 & 27.191 & 0.039 \\
\hline $1 \quad 11$ & 111 & 17 & 0.019 & -0.002 & 27.218 & 0.055 \\
\hline 1 | 1 & 101 & 18 & 0.067 & 0.107 & 27.577 & 0.069 \\
\hline 1 I 1 & 1 1 1 & 19 & -0.022 & 0.098 & 27.616 & 0.091 \\
\hline $1 \quad 11$ & I & 20 & 0.055 & 0.165 & 27.876 & 0.112 \\
\hline
\end{tabular}

Fuente: Elaboración propia con datos de CNBV e INEGI

Para las pruebas de raíz unitaria se supone una serie de tiempo, $Y_{t}$, que puede ser representada como $Y_{t}=\beta_{1} Y_{t-1}+u_{t}$ (42), donde $u_{t}$ es conocido como ruido blanco, con media cero varianza constante y no esta correlacionado. Si se resta $Y_{t-1}$ de ambos miembros de la igualdad se obtiene $\Delta \mathrm{Y}_{t}=\delta \mathrm{Y}_{t-1}+u_{t},-1 \leq \delta \leq 1$ donde $\delta=\beta_{1}-1$ y $\Delta Y_{t}=Y_{t}-Y_{t-1}$ es el operador de primeras diferencias. Por probar que $\delta=0$, lo que implica que $\beta_{1}=1$, entonces se dice que la serie posee raíz unitaria, es decir, no es estacionaria. Las hipótesis son $H_{0}: \delta=0$ existe una raíz unitaria, es decir, la serie de tiempo es no estacionaria, y $H_{1}: \delta<0$ la serie de tiempo es estacionaria. Una forma más general es la prueba $\mathrm{ADF}$, porque considera que el término de error $u_{t}$ está correlacionado. La prueba Phillips-Perron (PP) utiliza métodos estadísticos no paramétricos, con el fin de evitar la correlación serial en los términos de error en la ecuación $\Delta Y_{t}=\beta_{1}+\beta_{2} t+\delta Y_{t-1}+u_{t}$. Existen tres posibilidades de que una caminata aleatoria pueda ser modelada: 1) que no tenga intercepto, 2) que tenga intercepto o 3) que tenga tendencia. Esta información es importante para aplicar las pruebas ADF y PP que confirmarán o rechazarán la presencia de raíz unitaria. En el Cuadro 6 se representan cada una de estas posibilidades para las variables de estudio. 


$\begin{array}{ll} & \\ \text { Caminata aleatoria } & \Delta \mathrm{r}_{-} \mathrm{IGAE}_{t}=\delta \mathrm{r}_{-} \mathrm{IGAE}_{t-1}+u_{t} \\ \text { Caminata aleatoria con intercepto } & \Delta \mathrm{r}_{-} \mathrm{IGAE}_{t}=\beta_{0}+\delta \mathrm{r}_{-} \mathrm{IGAE}_{t-1}+u_{t} \\ \text { Caminata aleatoria con tendencia determinista } & \Delta \mathrm{r}_{-} \mathrm{IGAE}_{t}=\beta_{0}+\beta_{2} t+\delta \mathrm{r}_{-} \mathrm{IGAE} \mathrm{G}_{t-1}+u_{t}\end{array}$

$r_{-} B b v a_{t}$

Caminata aleatoria

Caminata aleatoria con intercepto

Caminata aleatoria con tendencia determinista

$r_{-}$Banorte $_{t}$

Caminata aleatoria

Caminata aleatoria con intercepto

Caminata aleatoria con tendencia determinista

$$
\begin{aligned}
\Delta \mathrm{r}_{-} \text {Banorte }_{t} & =\delta \mathrm{r}_{-} \text {Banorte }_{t-1}+u_{t} \\
\Delta \text { r_Banorte }_{t} & =\beta_{0}+\delta \mathrm{r}_{-} \text {Banorte }_{t-1}+u_{t} \\
\Delta \mathrm{r}_{-} \text {Banorte }_{t} & =\beta_{0}+\beta_{2} t+\delta \mathrm{r} \_B a n o r t e_{t-1}+u_{t}
\end{aligned}
$$

Fuente: Elaboración propia con datos de CNBV e INEGI

Para las pruebas ADF y PP, las hipótesis son las mismas. No se rechaza la hipótesis nula $H_{0}$ de la presencia de raíz unitaria si el valor absoluto de la estadística $t$ no excede cualquiera de los valores críticos. Esto es $\mid$ Estadístico (ADF y PP) $|<|$ Valores críticos (1.5 y 10\%)|. Otro elemento a considerar es la probabilidad $p$. Si $p>0.01,0.05$ ó 0.10 implica que no se rechaza la hipótesis nula $H_{0}$ con un nivel de confianza del 99\%, $95 \%$ y del $90 \%$, respectivamente. En caso contrario se rechaza $H_{0}$ y no existe raíz unitaria, lo cual indica que la serie es estacionaria. A continuación se muestran las pruebas de raíz unitaria de las series de tiempo. Primero se determina el proceso generador de datos (PGD), para ello se toman en cuenta las Gráfica 1, 2 y 3, y se observa que se trata de caminatas aleatorias con intercepto.

El Cuadro 7 presenta los resultados de las pruebas ADF y PP para la serie r_IGAE. Al 1\%, 5\% y 10\% se rechaza la hipótesis nula. Es decir, r_IGAE no tiene presencia de raíz unitaria, por lo que es estacionaria, ya que el valor de la estadística de prueba en valor absoluto es mayor a los valores críticos en valor absoluto y además el valor pes menor que los niveles de significación del 0.01, 0.05 y 0.10 . 
Prueba ADF y PP de r_IGAE

\begin{tabular}{|c|c|c|c|c|c|}
\hline & & \multicolumn{2}{|c|}{ Dickey-Fuller Aumentada } & \multicolumn{2}{|c|}{ Phillips-Perron } \\
\hline & & Estadística $t$ & Prob * & Estadística $t$ ajustada & Prob * \\
\hline \multicolumn{2}{|c|}{ Estadística de prueba } & -5.3721 & 0.0000 & -5.3924 & 0.0000 \\
\hline Valores & $1 \%$ & -3.5812 & & -3.5812 & \\
\hline críticos de & $5 \%$ & -2.9266 & & -2.9266 & \\
\hline la prueba: & $10 \%$ & -2.6014 & & -2.6014 & \\
\hline
\end{tabular}

* MacKinnon (1996) one-sided p-values.

Fuente: Elaboración propia con datos de CNBV e INEGI

El Cuadro 8 muestra los resultados de las pruebas ADF y PP para la serie r_Bbva con intercepto. Se rechaza la hipótesis nula, la serie no presenta raíz unitaria, por lo cual r_Bbva es estacionaria ya que al 1\%, 5\% y $10 \%$ el valor del estadístico en valor absoluto es mayor a los valores críticos y el valor de $p$ es menor que el nivel de significancia correspondiente al 0.01, 0.05 y 0.10. Esto implica que los rendimientos de Bbva son estacionarios.

\begin{tabular}{lccccc}
\hline & & \multicolumn{2}{c}{ Dickey-Fuller Aumentada } & \multicolumn{2}{c}{ Phillips-Perron } \\
& & Estadística $t$ & Prob ${ }^{*}$ & Estadística $t$ ajustada & Prob $^{*}$ \\
\hline Estadística de prueba & -7.8676 & 0.0000 & -7.8578 & 0.0000 \\
Valores & $1 \%$ & -3.5812 & & -3.5812 & \\
críticos de & $5 \%$ & -2.9266 & & -2.9266 & \\
la prueba: & $10 \%$ & -2.6014 & & -2.6014 \\
\hline
\end{tabular}

* MacKinnon (1996) one-sided p-values.

Fuente: Elaboración propia con datos de CNBV e INEGI

El Cuadro 9 presenta los resultados de las pruebas ADF y PP para r_Banorte. Dado que el valor del estadístico en valor absoluto es mayor a los valores críticos y el valor de $p$ es menor que los niveles de significación del $0.01,0.05$ y 0.10 , entonces al 1\%, 5\% y 10\% se rechaza la hipótesis nula, es decir, la serie r_Banorte no tiene presencia de raíz unitaria, por lo que es estacionaria. 


\begin{tabular}{|c|c|c|c|c|c|}
\hline & & \multicolumn{2}{|c|}{ Dickey-Fuller Aumentada } & \multicolumn{2}{|l|}{ Phillips-Perron } \\
\hline & & Estadística $t$ & Prob * & Estadística $t$ ajustada & Prob * \\
\hline \multicolumn{2}{|c|}{ Estadística de prueba } & -11.2002 & 0.0000 & -11.0595 & 0.0000 \\
\hline Valores & $1 \%$ nivel & -3.5812 & & -3.5812 & \\
\hline críticos de & $5 \%$ nivel & -2.9266 & & -2.9266 & \\
\hline la prueba: & $10 \%$ nivel & -2.6014 & & -2.6014 & \\
\hline
\end{tabular}

* MacKinnon (1996) one-sided p-values.

Fuente: Elaboración propia con datos de CNBV e INEGI

Una vez que se verificó que las series r_IGAE, r_Bbva y r_Banorte son estacionarias, el siguiente paso es determinar el valor del rezago $p$ de los procesos $\operatorname{AR}(p)$ de cada serie. Se dice que la serie $Y_{t}$ sigue un proceso autorregresivo de orden $p, \operatorname{AR}(p)$, si se puede representar como $Y_{t}=\beta_{0}+\beta_{1} Y_{t-1}+\beta_{1} Y_{t-1}+\cdots+\beta_{p} Y_{t-p}+u_{t}$. Es decir, la serie $Y_{t}$ se puede expresar en términos de sus valores pasados más una es útil para identificar el proceso que mejor se ajusta a los datos, se puede resumir en tan sólo cuatro pasos. Siguiendo la metodología de BoxJenkins, el primer paso a realizar es el de identificación (antes ya presentado en esta investigación); una de las herramientas más importantes y empleadas son los correlogramas de cada una de las serie de tiempo, los cuales ayudarán a identificar el valor del rezago $p$ apropiado para estimar el PGD. El Cuadro 10 presenta las características de las FAC y FAP para considerar el proceso $\mathrm{AR}(p)$ a emplear.

Cuadro 10 Características de las FAC y FAP para determinar el proceso AR(p)

\begin{tabular}{lll}
\hline Procesos & \multicolumn{1}{c}{ FAC } & FACP \\
\hline \multirow{2}{*}{$\mathrm{AR}(1)$} & Decae rápidamente a cero, o con forma & Desaparece después del \\
& sinusoidal o una combinación de estos. & rezago $q$ \\
& & \\
$\operatorname{AR}(p)$ & Decae rápidamente a cero, los coeficientes & Un pico en $q$ \\
& pueden oscilar. & \\
&
\end{tabular}


Con base en la información del Cuadro 10, el correlograma y el correlograma parcial de la serie r_IGAE que se muestran en la Gráfica 4, se puede observar que el proceso AR(4) es candidato a ser el PGD, ya que el diagrama del correlograma tiene una FACP que decrece en los primeros tres rezagos, los cuales son estadísticamente no significativos, salvó el cuarto rezago. También se puede sugerir como un modelo alternativo un proceso $\mathrm{AR}(4)$ o $\mathrm{AR}(6)$, ya que el sexto rezago también es estadísticamente diferente de cero. Los modelos sugeridos son:

Modelo 1: $\operatorname{AR}(4)$

Modelo 2: $\operatorname{AR}(6)$

$$
\text { r_IGAE }{ }_{t}=\beta_{0}+\beta_{4} \mathrm{r}_{-} \mathrm{IGAE}_{t-4}+u_{t}
$$$$
\mathrm{r}_{-} \mathrm{IGAE}_{t}=\beta_{0}+\beta_{4} \mathrm{r}_{-} \mathrm{IGAE}_{t-4}+\beta_{6} \mathrm{r}_{-} \mathrm{IGAE}_{t-6}+u_{t}
$$

El siguiente paso es la estimación de los parámetros. Los resultados se pueden ver en el Cuadro 11 para el modelo 1 y en el Anexo 5 para el modelo 2. Al comparar los resultados de ambos modelos se deduce, hasta el momento, que el modelo 1 es el que mejor se ajusta a los datos. Además, el estimador del parámetro $\beta_{4}$ es estadísticamente significativo para ambas modelos, los otros parámetros estimados no lo son.

Cuadro 11

Estimación modelo AR(4) para r_IGAE

\begin{tabular}{lcccc}
\hline Variable & Coeficiente & Error Estándar & Estadística $t$ & Prob. \\
\hline C & 0.000654 & 0.001932 & 0.338582 & 0.7367 \\
AR(4) & 0.420858 & 0.140177 & 3.002334 & 0.0045 \\
& & & -6.9482 \\
criterio de información de Akaike & & & -6.8663 \\
criterio de Schwarz & & & \\
\hline
\end{tabular}

Fuente: Elaboración propia con datos de CNBV e INEGI

El último paso es el examen del diagnóstico que consiste en analizar los residuos de ambos modelos. El propósito es indagar si los residuos son ruido blanco con apoyo del correlograma y el gráfico de residuos. En el caso del correlograma se tiene que contemplar que las autocorrelaciones de los rezagos no son estadísticamente significativos. En otras palabras, la FAC y la FACP no salen de las bandas de confianza (las líneas punteadas que se encuentran a ambos lados de las barras) y que la Prob sea superior a 0.05 para que sea confiable en un $95 \%$.

En la Gráfica 7 se aprecia el correlograma del modelo 1, se pueden ver la autocorrelaciones y las autocorrelaciones parciales, y se observa que no son estadísticamente significativas de manera individual, salvo el rezago 6 de la FACP que está en el límite de la banda de confianza, tampoco lo es la suma de las autocorrelaciones elevadas al cuadrado, en otras palabras, los residuos estimados de la Gráfica 7 son ruido blanco al 95\% de confianza ${ }^{9}$.

\footnotetext{
${ }^{9} \mathrm{El}$ correlograma del modelo 2 se puede consultar en Anexo 5. Al igual que en el modelo 1 se concluye que los residuos son ruido blanco pero por los criterios de información se eligió el modelo 1.
} 


\begin{tabular}{|c|c|c|c|c|c|c|c|c|}
\hline \multicolumn{2}{|c|}{ Autocorrelation } & \multicolumn{3}{|c|}{ Partial Correlation } & \multirow{2}{*}{$\frac{A C}{0.115}$} & \multirow{2}{*}{$\frac{P A C}{0.115}$} & \multirow{2}{*}{$\frac{\text { Q-Stat }}{0.6125}$} & \multirow[t]{2}{*}{ Prob } \\
\hline 1 & 口 I & . & & 1 & & & & \\
\hline 1 & $\mathbf{n}_{1}$ & $1=$ & & 2 & 0.228 & 0.218 & 3.0656 & 0.080 \\
\hline 1 & $\mathbf{b}_{1}$ & $1=$ & & 3 & 0.236 & 0.202 & 5.7511 & 0.056 \\
\hline 1 I & 1 , & 1 & । & 4 & 0.057 & -0.025 & 5.9106 & 0.116 \\
\hline 1 I & 1 & 11 & । & 5 & 0.111 & 0.018 & 6.5337 & 0.163 \\
\hline ( & 1 & ש & 1 & 6 & -0.237 & -0.330 & 9.4785 & 0.091 \\
\hline , & 1 & (1) & 1 & 7 & -0.205 & -0.258 & 11.739 & 0.068 \\
\hline I I & 1 & 1 I & 1 & 8 & -0.049 & 0.052 & 11.869 & 0.105 \\
\hline , I & 1 & $1 \quad$ E & 1 & 9 & -0.096 & 0.168 & 12.398 & 0.134 \\
\hline | I & 1 & 1 I & 1 & 10 & -0.074 & 0.107 & 12.719 & 0.176 \\
\hline , & 1 & , & । & 11 & 0.181 & -0.141 & 14.705 & 0.143 \\
\hline I I & 1 & , & 1 & 12 & -0.043 & -0.148 & 14.822 & 0.191 \\
\hline 11 & 1 & 1 & 1 & 13 & -0.009 & -0.134 & 14.827 & 0.251 \\
\hline 10 & 1 & 11 & 1 & 14 & -0.057 & -0.016 & 15.048 & 0.304 \\
\hline I I & 1 & 11 & 1 & 15 & -0.085 & 0.032 & 15.543 & 0.342 \\
\hline , & 1 & I I & 1 & 16 & 0.166 & -0.092 & 17.523 & 0.289 \\
\hline 1 I & 11 & I I & 1 & 17 & 0.064 & 0.062 & 17.827 & 0.334 \\
\hline 11 & 1 & 11 & 1 & 18 & 0.025 & 0.062 & 17.875 & 0.397 \\
\hline I I & 1 & 1 & 1 & 19 & _. 0095 & _. 0085 & 18595 & 0417 \\
\hline I I & 1 & 1 & 1 & 20 & -0.030 & -0.129 & 18.671 & 0.478 \\
\hline
\end{tabular}

Fuente: Elaboración propia

Otro elemento elemento que corrobora la no autocorrelación residual es el gráfico de los residuos (ver anexo 7) dado que un gran número de residuos se encuentran dentro de las bandas de confianza. Por último, la representación del modelo que mejor ajusta los datos de r_IGAE es:

$$
\text { r_IGAE }_{t}=0.000654+0.420858 r_{-} I_{G A E}-4
$$

Para el caso de r_Bbva, su correlograma se muestra en la Gráfica 5 y se puede sugerir el proceso AR(1), como posible candidato para ser el PGD, porque la FACP muestra que el primer rezago es estadísticamente significativa, pero también lo es el rezago 11, por lo que también se puede considerar como modelos alternativos, los procesos $\operatorname{AR}(1) \operatorname{AR}(11)$ :

$$
\begin{array}{ll}
\text { Modelo 3:AR(1) } & \mathrm{r}_{-} \text {Bbva }_{t}=\beta_{0}+\beta_{1} \mathrm{r}_{\mathrm{Bbva}} t-1+u_{t} \\
\text { Modelo 4: } \operatorname{AR}(11) & \mathrm{r}_{-} \mathrm{Bbva}_{t}=\beta_{0}+\beta_{1} \mathrm{r}_{-} \text {Bbva }_{t-1}+\beta_{11} \mathrm{r}_{-} \text {Bbva }_{t-11}
\end{array}
$$

En el cuadro 12 se presentan los resultados del modelo 3; ver el Anexo 5 para el modelo 4 . Al comparar los resultados de ambos modelos se concluye que el modelo 3 es un candidato potencial, ya que los criterios de información, Akaike y Schwarz son mejores que los del modelo 3. Los estimadores de los parámetros son estadísticamente significativos para ambos modelos. Se puede ver en la Gráfica 8, que los residuos son ruido blanco para el modelo 3. 
Estimación modelo AR(1) para r_Bbva

\begin{tabular}{|c|c|c|c|c|}
\hline Variable & Coeficiente & Error Estándar & Estadística $t$ & Prob. \\
\hline C & 0.10387 & 0.041711 & 2.48028 & 0.0166 \\
\hline $\mathrm{AR}(1)$ & -0.45281 & 0.184658 & -2.45218 & 0.0182 \\
\hline \multicolumn{4}{|c|}{ criterio Akaike } & 1.009236 \\
\hline \multicolumn{4}{|c|}{ criterio Schwarz } & 1.141067 \\
\hline
\end{tabular}

Fuente: Elaboración propia con datos de CNBV e INEGI

Gráfica 8

\begin{tabular}{|c|c|c|c|c|c|c|}
\hline Autocorrelation & Partial Correlation & & $A C$ & $P A C$ & Q-Stat & Prob \\
\hline 111 & 111 & 1 & 0.019 & 0.019 & 0.0172 & \\
\hline 11 & 111 & 2 & -0.045 & -0.046 & 0.1197 & 0.729 \\
\hline , 口 1 & , I & 3 & -0.128 & -0.126 & 0.9595 & 0.619 \\
\hline 11 & 111 & 4 & -0.058 & -0.057 & 1.1345 & 0.769 \\
\hline 1 I 1 & 111 & 5 & 0.074 & 0.066 & 1.4325 & 0.839 \\
\hline 1 | 1 & 101 & 6 & -0.076 & _. 0.101 & 1.7527 & 0.882 \\
\hline 111 & 111 & 7 & 0.031 & 0.026 & 1.8089 & 0.936 \\
\hline , 口 । & , I । & 8 & -0.119 & -0.117 & 2.6255 & 0.917 \\
\hline 111 & 111 & 9 & -0.005 & -0.013 & 2.6268 & 0.956 \\
\hline , 口 । & । & 10 & _. 0.174 & . 0.203 & 4.4882 & 0.876 \\
\hline । 口 । & । & 11 & -0.162 & 0.186 & 6.1431 & 0.803 \\
\hline 10 & 10 & 12 & 0.327 & 0.310 & 13.070 & 0.289 \\
\hline I I I & , । & 13 & _. 0.048 & _. 0.136 & 13.224 & 0.353 \\
\hline 111 & 111 & 14 & 0.010 & _. 0.051 & 13.231 & 0.430 \\
\hline 1 I 1 & 111 & 15 & -0.058 & 0.041 & 13.474 & 0.490 \\
\hline ） । & । & 16 & -0.122 & 0.180 & 14.564 & 0.483 \\
\hline 111 & 111 & 17 & 0.025 & - 0.040 & 14.610 & 0.553 \\
\hline 111 & 111 & 18 & -0.004 & 0.003 & 14.611 & 0.623 \\
\hline 1 ! 1 & 111 & 19 & 0.115 & 0.019 & 15.696 & 0.614 \\
\hline 1 I 1 & 111 & 20 & -0.038 & _. 0.017 & 15.818 & 0.669 \\
\hline
\end{tabular}

Fuente: Elaboración propia con datos de CNBV e INEGI

El gráfico de los residuos corrobora la ausencia de autocorrelación residual (ver anexo 7) porque la mayoría de los residuos se encuentran dentro de las bandas de confianza. Para terminar este estudio, la representación que mejor se ajusta a los datos de r_Bbva es:

$$
\mathrm{r}_{-} \text {Bbva }_{t}=0.10387-0.45281 \mathrm{r}_{-} \text {Bbva }_{t-1}
$$


El correlograma de r_Banorte se muestra en la Gráfica 6 y se puede suponer que el proceso AR(1) es un candidato para ser el PGD ya que el diagrama FACP muestra que el primer rezago no es estadísticamente significativo El modelo propuesto es representado como:

$$
\text { Modelo } 5 \operatorname{AR}(1) \quad r_{-} \text {Banorte }_{t}=\beta_{0}+\beta_{1} \mathrm{r}_{-} \text {Banorte }_{t-1}+u_{t}
$$

El Cuadro 13 presenta los resultados del modelo 5, con los cuales se concluye que el modelo 5 es un candidato potencial ya que los coeficientes de los parámetros son estadísticamente significativos. El modelo 5 presenta residuales que son ruido blanco, lo cual se observa en la Gráfica 9

\section{Cuadro $13 \quad$ Estimación modelo AR(1) para r_Banorte}

\begin{tabular}{|c|c|c|c|c|}
\hline Variable & Coeficiente & Error Estándar & Estadísticat & Prob. \\
\hline C & 0.18778 & 0.049902 & 3.762905 & 0.0005 \\
\hline $\operatorname{AR}(1)$ & -0.46276 & 0.130601 & -3.54332 & 0.0009 \\
\hline \multicolumn{3}{|c|}{ criterio de información Akaike } & & 1.473882 \\
\hline \multicolumn{3}{|c|}{ criterio de Schwarz } & & 1.553388 \\
\hline
\end{tabular}

Fuente: Elaboración propia con datos de CNBV e INEGI

Correlograma de los residuos del modelo 5

\begin{tabular}{|c|c|c|c|c|c|c|}
\hline Autocorrelation & Partial Correlation & & $A C$ & $P A C$ & Q-Stat & Prob \\
\hline 111 & 111 & 1 & -0.027 & -0.027 & 0.0353 & \\
\hline 111 & $1 \quad 1 \quad 1$ & 2 & 0.029 & 0.028 & 0.0777 & 0.780 \\
\hline $1 \theta_{1}$ & $1 \quad$ ， & 3 & 0.134 & 0.136 & 1.0006 & 0.606 \\
\hline E 1 & E । & 4 & -0.295 & -0.294 & 5.5754 & 0.134 \\
\hline $1 \quad 1$ & $1 \quad 11$ & 5 & 0.016 & 0.002 & 5.5886 & 0.232 \\
\hline 101 & । 1 & 6 & -0.166 & -0.181 & 7.1096 & 0.213 \\
\hline 11 & 111 & 7 & -0.079 & 0.001 & 7.4606 & 0.280 \\
\hline 101 & । & 8 & -0.096 & -0.207 & 7.9918 & 0.333 \\
\hline 1 & , 口 1 & 9 & -0.183 & -0.153 & 9.9978 & 0.265 \\
\hline $1 \quad 1$ & 101 & 10 & 0.002 & -0.121 & 9.9981 & 0.351 \\
\hline 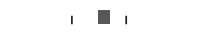 & । $\quad$ । & 11 & 0.116 & 0.148 & 10.845 & 0.370 \\
\hline 1 I 1 & 111 & 12 & 0.088 & 0.012 & 11.343 & 0.415 \\
\hline 111 & 1 ! & 13 & 0.003 & -0.113 & 11.344 & 0.500 \\
\hline $1 \quad$ - & $1 \quad 1 \quad 1$ & 14 & 0.122 & 0.008 & 12.376 & 0.497 \\
\hline 111 & 1 & 15 & 0.067 & 0.087 & 12.696 & 0.551 \\
\hline 11 & 11 & 16 & -0.045 & -0.041 & 12.842 & 0.615 \\
\hline 101 & 1 | 1 & 17 & 0.125 & 0.074 & 14.024 & 0.597 \\
\hline 1 1 & । $\mathbf{E}_{1}$ & 18 & 0.113 & 0.168 & 15.037 & 0.593 \\
\hline 111 & $1 \quad$ ， & 19 & 0037 & 0153 & 15150 & 0652 \\
\hline 111 & $1 \mathbf{1}_{1}$ & 20 & 0.049 & 0.128 & 15.352 & 0.700 \\
\hline
\end{tabular}

Fuente: Elaboración propia con datos de CNBV e INEGI 
El gráfico de los residuos corrobora la ausencia de autocorrelación residual (ver anexo 7). Por lo tanto, la representación del modelo que mejor se ajusta a los datos de r_Banorte es:

$$
\text { r_Banorte }{ }_{t}=0.18777-0.46276 r_{-} \text {Banorte }_{t-1}
$$

Los procesos AR estimados se usarán para obtener el modelo Markov Switching (MS) con dos regímenes, expansión y contracción, para las estimaciones conjuntas de r_IGAE con r_Bbva (MS1) y el r_IGAE con r_Banorte (MS2)

\subsection{Modelo econométrico Markov Switching (MS)}

Una de las motivaciones por la que se eligió esté enfoque es porque los cambios de régimen expresados en un modelo lineal que englobe todos los cambios, sobre toda la muestra, podría no ser apropiado, además, el modelo general no es lineal, pero sí lo es en cada componente (Brooks, 2008). En seguida se aplica el enfoque Markov Swithching a las variables de interés empleando el software Eviews (versión 8). Para ello se establece como variable explicativa r_IGAE y variable explicada r_Bbva para el modelo MS1 y r_Banorte para el modelo MS2.

El Cuadro 14 muestra los resultados obtenidos al aplicar el método MS, en donde se comprueba que en efecto existen dos regímenes con sus respectivos parámetros, aunque la variable AR(1) no es estadísticamente significativa en el régimen 2. Es importante aclarar que el modelo MS1 estimado tiene como periodo de tiempo de marzo del 2007 a diciembre del 2009, ya que para un mayor periodo los resultados arrojaron que el modelo MS1 no era estacionario, esta información se puede consultar en el anexo 8.

Cuadro 14

\begin{tabular}{lcccc}
\hline Variable & Coeficiente & Error Estándar & Estadístico z & Prob. \\
& & Régimen 1 Expansión & \\
AR(1) & -1.24912 & 0.12877 & -9.70050 & 0.00000 \\
C & 0.68909 & 0.07664 & 8.99096 & 0.00000 \\
& & Régimen 2 Contracción & \\
AR(1) & 0.07663 & 0.17308 & 0.44274 & 0.65800 \\
C & 0.06992 & 0.03693 & 1.89361 & 0.05830 \\
\hline
\end{tabular}

Modelo MS1

Fuente: Elaboración propia con datos de CNBV e INEGI

Por lo tanto, los modelos se pueden expresar como:

$\begin{array}{ll}\text { Régimen 1: expansión: } & \mathrm{r}_{-} \mathrm{Bbva}_{t}=0.68909-1.24912 \mathrm{r}_{-} \mathrm{Bbva}_{t-1}+u_{t} \\ \text { Régimen 2: contracción: } & \mathrm{r}_{-} \mathrm{Bbva}_{t}=0.06992+0.07663 \mathrm{r}_{-} \mathrm{Bbva}_{t-1}+u_{t}\end{array}$ 
La matriz de probabilidades de transición que sigue un proceso de cadena de Markov es de 2 × 2, donde las variables de estado son $S_{1}=1$ (expansión) y $S_{2}=2$ (contracción). En el Cuadro 15 se presentan las probabilidades de transición.

Matriz de probabilidades de transición del modelo MS1

\begin{tabular}{lcc}
\hline & Expansión & Contracción \\
\hline Expansión & 0.33015 & 0.66985 \\
Contracción & 0.20697 & 0.79304 \\
\hline
\end{tabular}

Fuente: Elaboración propia con datos de CNBV e INEGI

La Gráfica 10 muestra las probabilidades filtradas de los estados para el modelo MS. El Cuadro 16 muestra la duración esperada de estar en cada uno de los estados, para el estado 1 (expansión) la duración es de 1.49287 periodos mensuales. Mientras que, para el estado 2 (contracción) es de 4.83173 periodos.
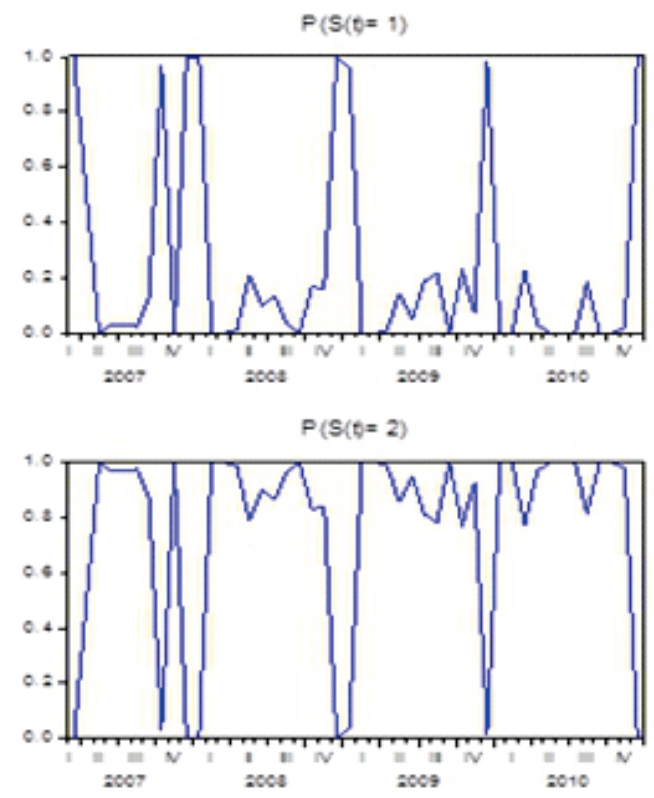

Fuente: Elaboración propia con datos de CNBV e INEGI 

Duración esperada de estar en el estado de expansión y contracción MS1

\begin{tabular}{lcc}
\hline & Expansión & Contracción \\
\hline 1.49287 & 4.83173 \\
\hline
\end{tabular}

Fuente: Elaboración propia con datos de CNBV e INEGI

El Cuadro 17 muestra los parámetros obtenidos del modelo MS2, se concluye que en efecto existe la presencia de dos estados y que los parámetros son estadísticamente significativos.

Cuadro 17

\begin{tabular}{lcccc}
\hline Variable & Coeficiente & Error Estándar & Estadístico z & Prob. \\
& & Régimen 1 Expansión & \\
AR(1) & -0.59951 & 0.16513 & -3.63059 & 0.00030 \\
C & 1.23677 & 0.14546 & 8.50280 & 0.00000 \\
& & Régimen 2 Contracción & \\
AR(1) & -0.50509 & 0.11131 & -4.53777 & 0.00000 \\
C & 0.12897 & 0.05628 & 2.29148 & 0.02190 \\
\hline
\end{tabular}

Fuente: Elaboración propia con datos de CNBV e INEGI

Considere ahora:

Régimen 1: expansión: $\quad r_{\_}$Banorte $_{t}=1.23677-0.59951 \mathrm{r}_{-}$Banorte $_{t-1}+u_{t}$

Régimen 2: contracción $\quad r_{-}$Banorte $_{t}=0.12897-0.50509 \mathrm{r}_{-}$Banorte $_{t-1}+u_{t}$

El Cuadro 18 muestra las probabilidades de transición. El Cuadro 19 muestra la duración esperada de estar en cada uno de los estados, para el estado 1 la duración es de 1.291246 periodos, mientras que para el estado 2 es de 13.37574 periodos. La Gráfica 11 muestra las probabilidades filtradas de los estados para el modelo MS2.

Cuadro 18

Matriz de probabilidades de transición del modelo MS2

\begin{tabular}{lcc}
\hline & Expansión & Contracción \\
\hline Expansión & 0.30060 & 0.69941 \\
Contracción & 0.12206 & 0.87794 \\
\hline
\end{tabular}

Fuente: Elaboración propia con datos de CNBV e INEGI 
Cuadro 19 Duración esperada de estar en el estado de expansión y contracción MS2

\begin{tabular}{lcc}
\hline Expansión & Contracción \\
\hline 1.42979 & 8.19249 \\
\hline
\end{tabular}

Fuente: Elaboración propia con datos de CNBV e INEGI

\section{Gráfica 11}

(s)

Fuente: Elaboración propia con datos de CNBV e INEGI

\subsection{Calibración econométrica de Markov Switching}

El proceso de difusión del consumo de las reservas bancarias de capital de BBVA Bancomer y BANORTE se puede modelar por medio de una ecuación diferencial estocástica de la siguiente manera:

$$
\begin{aligned}
& \mathrm{dr}_{-} \mathrm{Bbva}_{t}=a_{i}\left(b_{i}-\mathrm{r}_{-} \mathrm{Bbva}_{t}\right) \mathrm{d} t+\sigma_{1} \mathrm{~d} W_{t} \\
& \mathrm{~d} r_{-} \text {banorte }_{t}=a_{i}\left(b_{i}-r_{-} \text {banorte }_{t}\right) \mathrm{d} t+\sigma_{2} \mathrm{~d} W_{t}
\end{aligned}
$$

Los parámetros pueden ser estimados utilizando el modelo MS. Las dos ecuaciones anteriores se pueden plantear en términos discretos como una ecuación diferencial estocástica en diferencias, al hacer $\beta_{0}=a b \quad$ y $\beta_{1}=1-a$ (Venegas-Martínez, 2008), entonces las ecuaciones se reescriben como: 


$$
\begin{aligned}
& \mathrm{r}_{-} \mathrm{Bbva}_{t}=\beta_{0}+\beta_{1} \mathrm{r}_{-} \mathrm{Bbva}_{t-1}+u_{t} \\
& r_{-} \text {banorte }_{t}=\beta_{0}+\beta_{1} r_{-} \text {banorte } e_{t-1}+u_{t}
\end{aligned}
$$

Se tienen dos estados para cada una de las variables, se determinaron los parámetros, en cada caso, esto con la finalidad de ocuparlos para la simulación del modelo del proceso de difusión. Retomando las expresiones obtenidas con el método MS1, se pueden plantear las ecuaciones diferenciales estocásticas en diferencias para cada uno de los estados, en el caso de BBVA, estado 1 (expansión) se tiene:

$$
\mathrm{r}_{-} \mathrm{Bbva}_{t}=0.68909-1.24912 \mathrm{r}_{-} \mathrm{Bbva}_{t-1}+u_{t}
$$

donde $\beta_{0}=0.68909=a b \quad$ y $\beta_{1}=-1.24912=1-a$. Lo cual implica que $a=2.24912 \quad$ y $b=0.30638$. Con los parámetros obtenidos se pueden reescribir la ecuación diferencial estocástica como:

$$
\mathrm{dr}_{-} \mathrm{Bbva}_{t}=2.24912\left(0.30638-\mathrm{r}_{-} B b v a_{t}\right) \mathrm{d} t+0.428644 \mathrm{~d} W_{t}
$$

Para el estado 2 (contracción) se plantea la ecuación diferencial estocástica como una ecuación en diferencias:

$$
\mathrm{r}_{-} \text {Bbva }_{t}=0.06992+0.07663 \mathrm{r}_{-} \mathrm{Bbva}_{t-1}+u_{t}
$$

donde $\beta_{0}=0.06992=a b$ y $\beta_{1}=0.07663=1-a$. Al resolver el sistema de ecuaciones lineales se tiene $a=0.92337$ y $b=0.91244$. Con los parámetros obtenidos se puede reescribir la ecuación diferencial estocástica como:

$$
\mathrm{dr}_{-} \mathrm{Bbva}_{t}=0.92337\left(0.91244-\mathrm{r}_{-} \mathrm{Bbva}_{t}\right) \mathrm{d} t+0.428644 \mathrm{~d} W_{t}
$$

Con la información que se obtuvo con el método MS2, se pueden plantear las ecuaciones diferenciales estocásticas en diferencias, para cada uno de los estados para el caso de BANORTE. Para el estado 1 se tiene:

r_Banorte $_{t}=1.23677-0.59951 \mathrm{r}_{-}$Banorte $_{t-1}+u_{t}$

donde $\beta_{0}=1.23677=a b$ y $\beta_{1}=-0.59951=1-a$. Así, $a=1.59951$ y $b=0.77322$. Con los parámetros obtenidos, se pueden reescribir la ecuación diferencial estocástica, como:

$$
\mathrm{dr}_{-} \text {Banorte }_{t}=1.59951\left(0.77322-\mathrm{r}_{-} \text {Banorte }_{t}\right) \mathrm{d} t+0.55883 \mathrm{~d} W_{t}
$$

Para el estado 2 se puede plantear la ecuación diferencial estocástica como una ecuación en diferencias como para el Régimen 2 (contracción):

$$
\text { r_Banorte }_{t}=0.12897-0.50509 \text { r_Banorte }_{t-1}+u_{t}
$$


donde $\beta_{0}=0.12897=a b$ y $\beta_{1}=-0.50509=1-a$. Así, $a=1.50501$ y $b=0.08569$. Con los parámetros obtenidos, se pueden reescribir la ecuación diferencial estocástica, como:

$\mathrm{dr} \_$Banorte $_{t}=1.50501\left(0.08569-\mathrm{r}_{-}\right.$Banorte $\left.t\right) \mathrm{d} t+0.55883 \mathrm{~d} W_{t}$

Con esta información se simulará el proceso de difusión para ambas variables.

\subsection{Simulación Monte Carlo para estimar el tiempo de paro}

El objetivo de esta sección es conocer el tiempo de paro de BBVA Bancomer y Banorte, el cual indicará cuantos periodos, es decir, meses, tienen que pasar para que se consuman sus reservas bancarias de capital. Para obtener el tiempo de paro se realizó el algoritmo de simulación, usando la herramienta VisualBasic, y se replicó diez mil veces, a este método se le conoce como Monte Carlo.

El algoritmo emplea las propiedades estadísticas de los modelos MS1 y MS2, es decir, las probabilidades de transición de cada estado, las cuales se obtuvieron en el apartado anterior y modela las ecuaciones diferenciales estocásticas para BBVA Bancomer y Banorte, en expansión y contracción con los parámetros que se calcularon en las ecuaciones en diferencias. El algoritmo que se utilizó para calcular el tiempo de paro se describe a continuación. El primer paso es generar números aleatorios uniformes independientes $U_{1}, U_{2} \sim U(0,1)$, y después aplicar las transformaciones de Box-Muller:

$$
Z_{1}=\sqrt{-2 \log \left(U_{1}\right)} \cos \left(2 \pi U_{2}\right) \quad \text { y } \quad Z_{2}=\sqrt{-2 \log \left(U_{1}\right)} \operatorname{sen}\left(2 \pi U_{2}\right) .
$$

Las variables aleatorias $Z_{1}$ y $Z_{2}$ son independientes e idénticamente distribuidas $N(0,1)$. Los datos que se necesitaron para la elaboración de la rutina, para fines de consulta, puede ser solicitada a los autores. Los parámetros empleados en la rutina se muestran en el Cuadro 20.

Cuadro 20

Parámetros empleados en la rutina

\section{BBVA}

Régimen 1

$\begin{array}{ll}a & 2.249124 \\ b & 0.306380173 \\ r_{0} & 0.601559596 \\ \sigma & 0.428644\end{array}$

Régimen 2

Simulaciones

$\begin{array}{ll}a & 0.923369 \\ b & 0.912437525 \\ r_{0} & 0.601559596 \\ \sigma & 0.428644\end{array}$

$\begin{array}{ll}10,000 & \\ p_{11} & 0.330151 \\ p_{22} & 0.793035 \\ p_{21} & 0.206965 \\ p_{12} & 0.669849 \\ \text { Reserva } & 79.36 \\ \text { Capital contable } & 156\end{array}$

BANORTE

1.599508

0.773221516

0.21531

0.55883

1.505088

0.085688013

0.21531

0.55883

Fuente: Elaboración propia con datos de CNBV e INEGI 
Con la información anterior y con la ecuación diferencial estocástica $\mathrm{d} r_{t}=a\left(b-r_{t}\right) \mathrm{d} t+s Z \sqrt{\mathrm{d} t}$ se obtiene al tiempo de paro. El movimiento browniano se remplazó por $Z \sqrt{\mathrm{d} t}$. Los Los resultados son: el tiempo de paro para Banorte es de 22.09, esto es, cada 22 meses Banorte se termina sus reservas bancarias de capital, el tiempo de paro para Bancomer es de 9.45, cada 9 meses consume sus reservas bancarias de capital.

\section{Conclusiones}

Se investigó un tema de relevancia actual, el cual tiene implicaciones directas en la regulación de las reservas bancarias, por la evidencia empírica encontrada. Para ello se calibró una ecuación diferencial estocástica con datos empíricos con el objetivo de estimar el tiempo en que las instituciones de banca múltiple BBVA Bancomer y BANORTE consumen el capital para afrontar posibles pérdidas.

Se implementó un modelo denominado Markov Switching porque no es la misma cantidad de créditos no cumplidos cuando ocurre una recesión que cuando no se experimenta una. Por lo tanto, se vinculó el modelo de Markov Switching con una variable económica, el IGAE, útil para indagar si existe o no una recesión. Así, lo primero que se estudia en esta investigación es como la actividad económica, medida a través del IGAE, impacta la cantidad de quitas y castigos que hay por incumplimientos en los bancos. Segundo, en esta investigación se observa que el ritmo de quitas y castigos no es el mismo cuando la economía está estable que cuando hay una recesión. Tercero, se aportó evidencia empírica que expone como la velocidad a la que consumen de capital los bancos no es la misma, por lo que se deben diferenciar en el sistema bancario: a) las políticas para dar créditos ya que los prestatarios no son homogéneos; y, b) las políticas de cobranza son distintas por lo que los bancos tienen una responsabilidad directa de cuánto van reservando y cuánto envían destinan a créditos incobrables.

Se propuso un modelo básicamente reducido que modela la velocidad a la que se va consumiendo las reservas bancarias y se planteó como un modelo de tiempo de paro. Con dicho modelo se pudo estimar en cuánto tiempo se rompe la barrera de reservas que está determinada por Basilea. Se presento evidencia empírica de que la volatilidad es una variable determinante de la velocidad a la que se consume el capital, porque se obtuvieron simulaciones que tardan 15 ó 20 pasos, mientras que otras tardan 4. Esto, depende de manera importante de la volatilidad, lo cual se encuentra en línea con la hipótesis.

El trabajo aporta evidencia empírica sobre la fragilidad del sistema bancario sobre la cantidad de periodos que tardan en agotar su capital. Entre más rápido un banco agote sus reservas, más frágil es. Por tanto, el modelo puede ser empleado para modelar fragilidad de cada una de las entidades del sistema bancario para obtener los distintos periodos en los bancos incurrirían en paro si no resguardan sus reservas bancarias. Por último se observa que el sistema bancario no es homogéneo como lo muestra la calibración del modelo que se especificó. El tiempo de paro no es homogéneo y por tanto una regulación para todas las instituciones bancarias, como lo es la de Basilea, tendría que considerar la heterogeneidad dentro de la banca. Una extensión de esta investigación consiste en analizar a la banca especializada, especialmente a la dedicada a segmentos vulnerables.

\section{Referencias}

Altman, E. (1968). Financial ratios, discriminant analysis and the prediction of corporate bankruptcy. Journal of Finance, 23(Sept.), 589-609.

Atiya, A. (2001). Bankruptcy prediction for credit risk using neural networks: A survey and new results. IEEE Transactions on Neural Networks. 12(4): 929-935 
Banxico. (2010, enero 1). Reglas para los requerimientos de capitalización de las Instituciones de Banca Múltiple y las Sociedades Nacionales de Crédito, Instituciones de Bamca de Desarrollo. Disponible en: http://www.banxico.org.mx/waFormulariosDGASF/ReglasCapitalizacion338_40.htm

Basel Committee (2010, December). Basel III: A global regulatory framework for more resilient banks and banking systems. Basel: Bank for International Settlements.

Basel Committee (2010, October). Calibrating regulatory minimun capital requirements and capital buffers: a top-down approach . Basel, Switzerland: Bank for International Settlements.

Basel Committee (2004, June). International Convergence of capital measurement and capital standards. Basel, Switzerland: Bank for International Settlements.

Basel Committee (2008, November 28). Range of practices and issues in economic capital modelling . Basel, Switzerland: Bank for International Settlements.

Basel Committee (2011, November). Basel III counterparty credit risk. Switzerland: Bank for International Settlements.

Basel Committee (2014, December). Revisions to the standardised approach for credit risk. Basel, Switzerland: Bank for International Settlements.

Brooks, C. (2008). Introductory Econometrics for Finance. UK: Cambridge University Press.

Caruana, J. (2010, Septiembre 15). Basilea III: hacia un sistema financiero más seguro. Banco de Pagos Internacionales,

Claramunt, M. M., Casanovas, M., y Caicedo, E. (2012). Modelo para la predicción de indicadores de riesgo de crédito mediante razones financieras usando modelos estructurales y modelos de datos de panel: aplicación al mercado español. Academia. Revista Latinoamericana de Administración. 50: 118-147.

CNBV (2016). Portafolio de información. Obtenido de http://portafolioinfdoctos.cnbv.gob.mx/Documentacion/ minfo/00_DOC_R1.pdf

Crouhy, M., Galai, D., and Mark, R. (2000). A comparative analysis of current credit risk models. Journal of Banking and Finance, 24(1-2): 59-117.

Cruz Aké, S, F. Venegas-Martínez y A. Ortiz-Ramírez (2010). Riesgo de crédito un enfoque de cópulas y valores extremos. Eseconomía, Revista de Estudios Económicos, Tecnológicos y Sociales, 27, 7-33.

Dunkel, J., and Weber, S. (2007). Efficient Monte Carlo methods for convex risk measures in portafolio credit risk models. Proceedings of the 2007 Winter Simulation Conference, 958-956.

Elizalde, A., y Repullo, R. (2004). Capital económico y capital regulatorio: un análisis de sus determinantes. Estabilidad Financiera, 7:141-158.

Enders, W. (2015). Applied econometric time series. Wiley Series in Probability and Statistics. University of Alabama.

Geske, R. (1977). The valuation of corporate liabilities as compound options. Journal of Financial and Quantitative Analysis, 12(4): 541-552

Greene, W. (2002). Econometric analysis. New York: Prentice Hall.

Hamilton, J. (2005, may 18). Regime-Switching Models. Department of Economics. University of California, San Diego. La Jolla, CA.

INEGI. (2016). Banco de información económica. Disponible en: http://www.inegi.org.mx/Sistemas/BIE/ Default.aspx? Topic $=0$ \&idserPadre $=10000200 \#$ D10000200

JP Morgan and Company. (1997). CreditMetrics. Nueva York: JP Morgan . 
Ley de Instituciones de Crédito. (2014, enero 10). Diario Oficial de la Federación. Estados Unidos Mexicanos.

Merton, R. (1974). On the pricing of the corporate debt: the risk structure of the interest rates. Journal of Finance, 29(2): 449-70.

Ramaswany, S. (2005). Simulated credit loss distribution. Journal of Portfolio Management. 31(4): 91-99.

SHCP, CNBV. (2014). Resolución que modifica las disposiciones de carácter general aplicables a las Instituciones de Crédito. Disponible en el Diario Oficial de la Federación: http://dof.gob.mx/nota_detalle.php?codigo=5 361350\&fecha $=24 / 09 / 2014$

Van Gestel, T., and Baesens, B. (2009). Credit risk management. New York: Oxford University Press Inc.

Venegas-Martínez, F. (2008). Riesgos financieros y económicos; productos derivados y decisiones económicas bajo incertidumbre. Segunda Edición. México: Cengage Leraning Editores 


\section{Anexos}

Anexo 1

Evolución de las variables en niveles

\section{Evolución del IGAE}

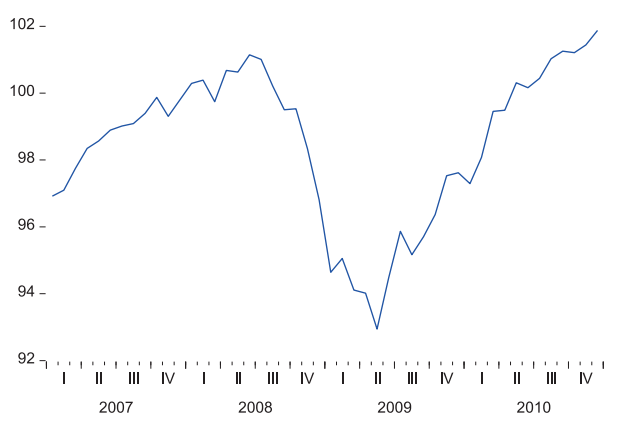

Evolución de BBVA

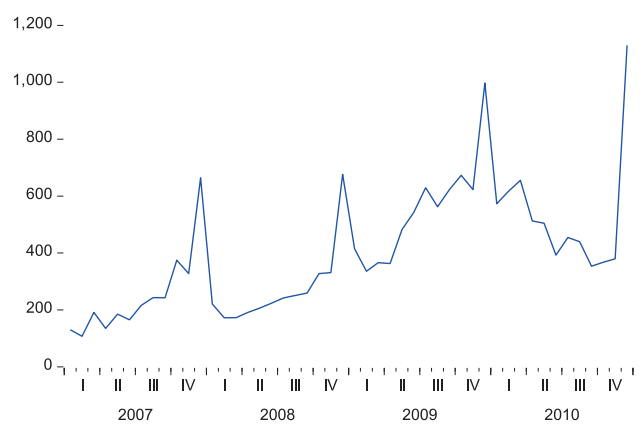

Evolución de Banorte

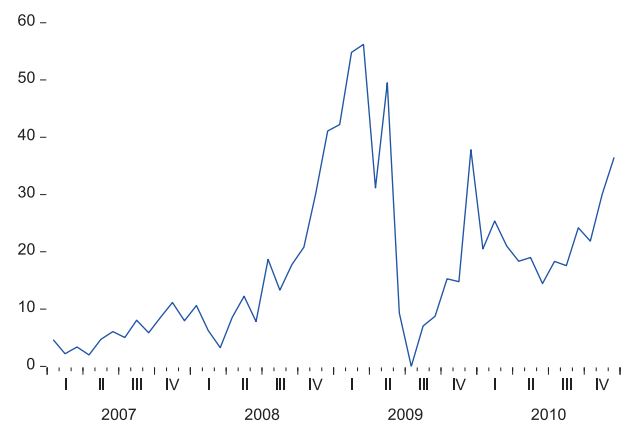

Fuente: Elaboración propia con datos de CNBV e INEGI 
Anexo 2

Correlogramas de las variables en niveles

Correlograma del IGAE

\begin{tabular}{|c|c|c|c|c|c|c|}
\hline Autocorrelation & Partial Correlation & & $A C$ & PAC & Q-Stat & Prob \\
\hline , & i & 1 & 0.919 & 0.919 & 43.152 & 0.000 \\
\hline 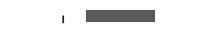 & , I & 2 & 0.822 & -0.151 & 78.370 & 0.000 \\
\hline 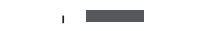 & 1 1 & 3 & 0.711 & 0.128 & 105.34 & 0.000 \\
\hline 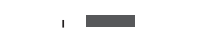 & i 1 & 4 & 0.584 & -0.161 & 123.95 & 0.000 \\
\hline ש & = । & 5 & 0.417 & -0.340 & 133.64 & 0.000 \\
\hline $\mathbf{n}_{1}$ & 101 & 6 & 0.243 & -0.147 & 137.02 & 0.000 \\
\hline I 1 & 111 & 7 & 0.086 & . 0.002 & 137.45 & 0.000 \\
\hline $1 \quad 1$ & $1 \quad 1 \quad 1$ & 8 & -0.054 & 0.000 & 137.63 & 0.000 \\
\hline I I & 111 & 9 & -0.190 & -0.066 & 139.86 & 0.000 \\
\hline - 1 & 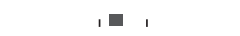 & 10 & .0 .324 & -0.161 & 146.47 & 0.000 \\
\hline । & 111 & 11 & 0.428 & .0 .058 & 158.36 & 0.000 \\
\hline ， & 111 & 12 & 0.490 & 0.074 & 174.34 & 0.000 \\
\hline ， & 101 & 13 & .0 .544 & . 0.124 & 194.61 & 0.000 \\
\hline ～， & I D 1 & 14 & 0.594 & _. 0.163 & 219.56 & 0.000 \\
\hline — & 1 | 1 & 15 & 0.622 & 0.094 & 247.67 & 0.000 \\
\hline — & 111 & 16 & .0 .610 & . 0.001 & 275.58 & 0.000 \\
\hline ए । & 1 ! 1 & 17 & .0565 & 0127 & 30030 & 0000 \\
\hline ， & 1 1 1 & 18 & .0 .523 & - 0.089 & 322.20 & 0.000 \\
\hline - & 111 & 19 & . 0.455 & 0.052 & 339.30 & 0.000 \\
\hline - & 1 1 1 & 20 & .0 .348 & 0.113 & 349.71 & 0.000 \\
\hline
\end{tabular}

Fuente: Elaboración propia con datos de CNBV e INEGI

Correlograma del BBVA

\begin{tabular}{|c|c|c|c|c|c|c|}
\hline Autocorrelation & Partial Correlation & & $A C$ & $P A C$ & Q-Stat & Prob \\
\hline $1 \square$ & 10 & 1 & 0.535 & 0.535 & 14.630 & 0.000 \\
\hline $1 \square$ & 10 & 2 & 0.492 & 0.288 & 27.267 & 0.000 \\
\hline 10 & , 口 , & 3 & 0.413 & 0.109 & 36.379 & 0.000 \\
\hline 10 & 111 & 4 & 0.361 & 0.056 & 43.468 & 0.000 \\
\hline 10 & 101 & 5 & 0.349 & 0.085 & 50.271 & 0.000 \\
\hline , $\boldsymbol{\sigma}_{1}$ & , I & 6 & 0.238 & -0.077 & 53.502 & 0.000 \\
\hline , & 111 & 7 & 0.249 & 0.045 & 57.127 & 0.000 \\
\hline , $\mathbf{D}_{1}$ & 11 & 8 & 0.171 & -0.046 & 58.885 & 0.000 \\
\hline , $\boldsymbol{D}_{1}$ & 11 & 9 & 0.182 & 0.040 & 60.919 & 0.000 \\
\hline , $\boldsymbol{~ 口 ~}_{\text {। }}$ & 11 & 10 & 0.146 & -0.004 & 62.266 & 0.000 \\
\hline , 口 , & 111 & 11 & 0.120 & -0.002 & 63.200 & 0.000 \\
\hline 10 & 10 & 12 & 0.391 & 0.442 & 73.416 & 0.000 \\
\hline , 口 । & E I & 13 & 0.139 & -0.281 & 74.746 & 0.000 \\
\hline $\mathbf{0}_{1}$ & , I & 14 & 0.120 & -0.152 & 75.766 & 0.000 \\
\hline 1 I 1 & , I & 15 & 0.041 & -0.098 & 75.889 & 0.000 \\
\hline 111 & I & 16 & -0.038 & -0.181 & 75.995 & 0.000 \\
\hline 1 I 1 & 11 & 17 & -0.029 & -0.035 & 76.061 & 0000 \\
\hline , I & 11 & 18 & -0.094 & 0.032 & 76.762 & 0.000 \\
\hline , I & 111 & 19 & -0.099 & -0.042 & 77.574 & 0.000 \\
\hline , 口 , & 11 & 20 & _. 0.177 & -0.035 & 80.254 & 0.000 \\
\hline
\end{tabular}

Fuente: Elaboración propia con datos de CNBV e INEGI 


\section{Correlograma de Banorte}

\begin{tabular}{|c|c|c|c|c|c|c|}
\hline Autocorrelation & Partial Correlation & & $A C$ & PAC & Q-Stat & Prob \\
\hline 1 & 1 & 1 & 0.718 & 0.718 & 26.360 & 0.000 \\
\hline 1 &,$\quad \infty$ & 2 & 0.688 & 0.356 & 51.085 & 0.000 \\
\hline , 口 & E , & 3 & 0.460 & -0.270 & 62.389 & 0.000 \\
\hline $1 \mathbf{a}_{1}$ & - I & 4 & 0.247 & -0.377 & 65.709 & 0.000 \\
\hline , & I $\mathbf{I}_{1}$ & 5 & 0.162 & 0.168 & 67.183 & 0.000 \\
\hline 111 & 1 I 1 & 6 & -0.002 & 0.053 & $67 \cdot 183$ & 0.000 \\
\hline 111 & , $\theta_{1}$ & 7 & 0.061 & 0.244 & 67.401 & 0.000 \\
\hline 111 & 101 & 8 & -0.008 & -0.101 & 67.404 & 0.000 \\
\hline 111 & 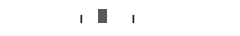 & 9 & 0.048 & -0.088 & 67.549 & 0.000 \\
\hline 111 & 111 & 10 & 0.072 & 0.025 & 67.877 & 0.000 \\
\hline 111 & 101 & 11 & 0.015 & -0.096 & 67.892 & 0.000 \\
\hline 111 & 111 & 12 & 0.042 & -0.024 & 68.009 & 0.000 \\
\hline 111 & 111 & 13 & -0.065 & -0.043 & 68.297 & 0.000 \\
\hline 101 & $1 \pm 1$ & 14 & -0095 & -0085 & 68928 & 0.000 \\
\hline 101 & 111 & 15 & -0.150 & 0.021 & 70.569 & 0.000 \\
\hline 101 & I $\quad$ I & 16 & -0.142 & 0.179 & 72.083 & 0.000 \\
\hline 101 & 101 & 17 & -0149 & -0120 & 73809 & 0000 \\
\hline 101 & 101 & 18 & -0.147 & -0.144 & 75.542 & 0.000 \\
\hline 101 & 111 & 19 & -0.095 & 0.020 & 76.296 & 0.000 \\
\hline 101 & 101 & 20 & -0.148 & -0.098 & 78.171 & 0.000 \\
\hline
\end{tabular}

Fuente: Elaboración propia con datos de CNBV e INEGI 
Anexo 3

Prueba de raíz unitaria de las variables en niveles

\begin{tabular}{|c|c|c|c|c|c|}
\hline \multicolumn{6}{|c|}{ Pruebas ADF y PP del IGAE } \\
\hline & & \multicolumn{2}{|c|}{ Dickey-Fuller Aumentada } & \multicolumn{2}{|c|}{ Phillips-Perron } \\
\hline & & Estadística $t$ & Prob * & Estadística $t$ ajustada & Prob * \\
\hline \multicolumn{2}{|c|}{ Estadística de prueba } & -0.7226 & 0.9653 & -1.2553 & 0.8867 \\
\hline Valores & $1 \%$ & -4.16576 & & -4.1658 & \\
\hline críticos de & $5 \%$ & -3.50851 & & -3.5085 & \\
\hline la prueba: & $10 \%$ & -3.18423 & & -3.1842 & \\
\hline
\end{tabular}

* MacKinnon (1996) one-sided p-values.

Fuente: Elaboración propia con datos de CNBV e INEGI

Pruebas ADF y PP de BBVA

\begin{tabular}{lccccc}
\hline & \multicolumn{2}{c}{ Dickey-Fuller Aumentada } & \multicolumn{2}{c}{ Phillips-Perron } \\
& \multicolumn{2}{c}{ Estadística $t$} & Prob $^{*}$ & Estadística $t$ ajustada & Prob $^{*}$ \\
\hline \multirow{2}{*}{ Estadística de prueba } & -2.2573 & 0.1898 & -1.9289 & 0.3166 \\
Valores & $1 \%$ & -3.57772 & -3.5777 & \\
críticos de & $5 \%$ & -2.92517 & -2.9252 \\
la prueba: & $10 \%$ & -2.60066 & -2.6007 \\
\hline
\end{tabular}

* MacKinnon (1996) one-sided $p$-values.

Fuente: Elaboración propia con datos de CNBV e INEGI

Pruebas ADF y PP de Banorte

\begin{tabular}{lccccc}
\hline & \multicolumn{2}{c}{ Dickey-Fuller Aumentada } & \multicolumn{2}{c}{ Phillips-Perron } \\
& & Estadística $t$ & Prob $^{*}$ & Estadística $t$ ajustada & Prob $^{*}$ \\
\hline \multirow{2}{*}{ Estadística de prueba } & -2.4153 & 0.1431 & -2.4847 & 0.1256 \\
Valores & $1 \%$ & -3.57772 & & -3.5777 & \\
críticos de & $5 \%$ & -2.92517 & -2.9252 \\
la Prueba: & $10 \%$ & -2.60066 & -2.6007 \\
\hline
\end{tabular}

* MacKinnon (1996) one-sided p-values.

Fuente: Elaboración propia con datos de CNBV e INEGI 
Anexo 4 Gráficas de la densidad Kernel
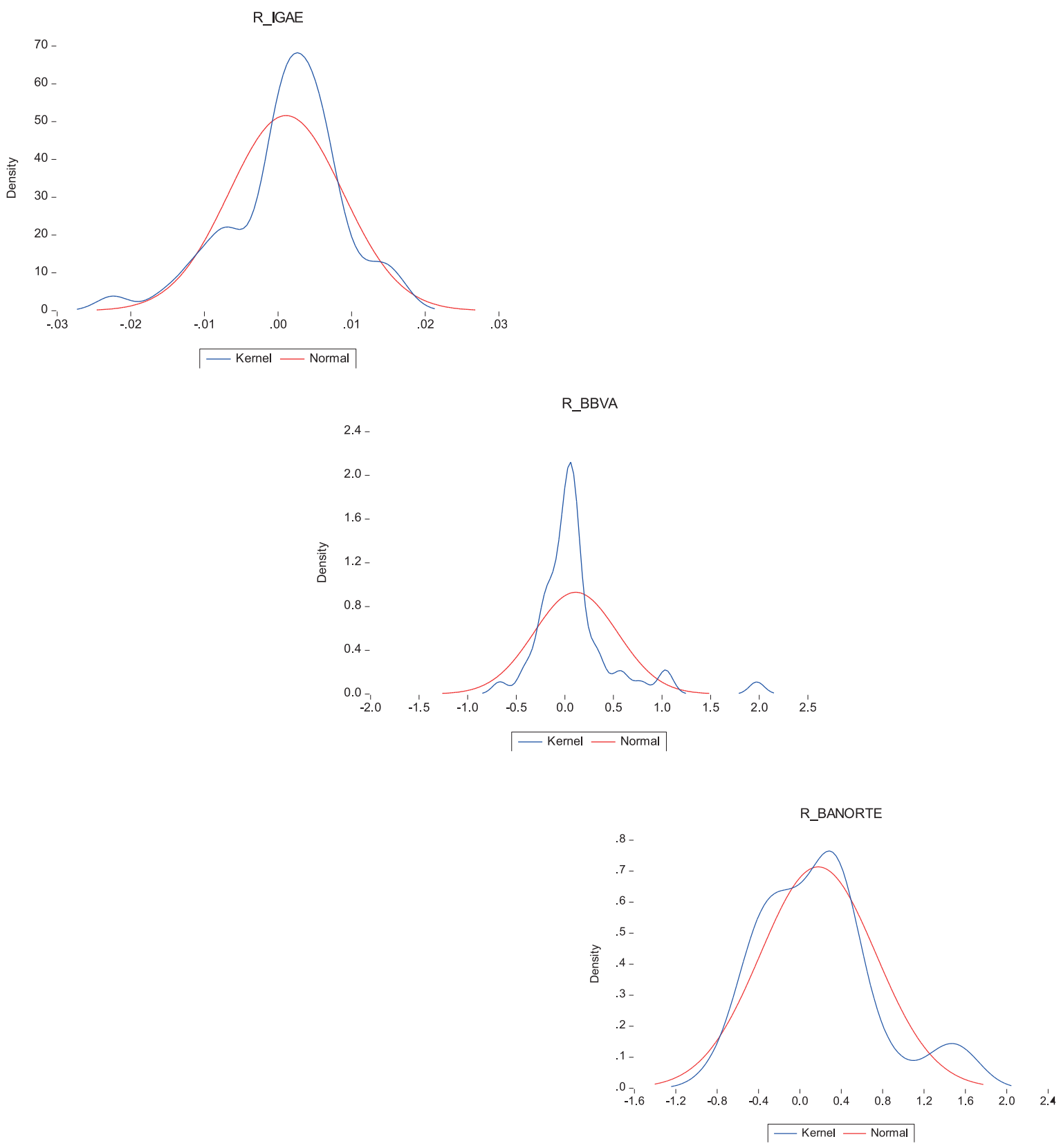

Fuente: Elaboración propia con datos de CNBV e INEGI 
Anexo 5

Resultados alternos de los procesos $\operatorname{AR}(p)$ para las variables de estudio

Modelo 2

\begin{tabular}{lcccc}
\hline Variable & Coeficiente & Error Estándar & Estadística $t$ & Prob. \\
\hline C & 0.000693 & 0.001507 & 0.45965 & 0.6484 \\
$\mathrm{AR}(4)$ & 0.464999 & 0.143938 & 3.230538 & 0.0026 \\
$\mathrm{AR}(6)$ & -0.22772 & 0.143747 & -1.58417 & 0.1214 \\
Criterio Akaike & & & -6.91525 \\
Criterio Schwarz & & & -6.78986 \\
\hline
\end{tabular}

Fuente: Elaboración propia con datos de CNBV e INEGI

Modelo 4

\begin{tabular}{llccc}
\hline Variable & Coeficiente & Error Estándar & Estadística $t$ & Prob. \\
\hline C & 0.078031 & 0.033179 & 2.351773 & 0.0248 \\
AR(1) & -0.43843 & 0.202504 & -2.16503 & 0.0377 \\
AR(11) & -0.50502 & 0.182427 & -2.76836 & 0.0092 \\
Criterio Akaike & & & 1.009433 \\
Criterio Schwarz & & & 1.141392 \\
\hline
\end{tabular}

Fuente: Elaboración propia con datos de CNBV e INEGI 


\begin{tabular}{|c|c|c|c|c|c|c|c|}
\hline Autocorr & relation & Partial Correlation & & $A C$ & $P A C$ & Q-Stat & Prob \\
\hline | I & 1 & 111 & 1 & 0.066 & 0.066 & 0.1946 & \\
\hline 1 & $\mathbf{a}$ & । $\quad$ | & 2 & 0.222 & 0.218 & 2.4176 & \\
\hline 1 & $\mathbf{a}$ & । $\square$ । & 3 & 0.272 & 0.260 & 5.8621 & 0.015 \\
\hline I I & 1 & $1 \quad 11$ & 4 & 0.043 & -0.021 & 5.9486 & 0.051 \\
\hline 1 & 1 & $1 \quad 1$ & 5 & 0.112 & -0.001 & 6.5620 & 0.087 \\
\hline । I & । & । 1 & 6 & -0.077 & -0.172 & 6.8624 & 0.143 \\
\hline । & । & 1 I & 7 & -0.201 & -0.268 & 8.9656 & 0.110 \\
\hline 11 & । & $1 \quad 1$ & 8 & -0.004 & 0.007 & 8.9664 & 0.175 \\
\hline I I & । & 1 I । & 9 & -0.062 & 0.121 & 9.1795 & 0.240 \\
\hline I I & I & 1 1 & 10 & -0.046 & 0.126 & 9.3002 & 0.318 \\
\hline । & I & 101 & 11 & -0.154 & -0.139 & 10.695 & 0.297 \\
\hline | I & I & 101 & 12 & -0.081 & -0.126 & 11.096 & 0.350 \\
\hline | I & I & 101 & 13 & -0.071 & -0.135 & 11.418 & 0.409 \\
\hline I I & 1 & 111 & 14 & -0.058 & -0.016 & 11.640 & 0.475 \\
\hline 1 & 1 & 111 & 15 & -0.106 & 0.009 & 12.407 & 0.495 \\
\hline I & 1 & I 1 & 16 & -0.231 & -0.150 & 16.157 & 0.304 \\
\hline 11 & 1 & 1 I 1 & 17 & 0.029 & 0.078 & 16.220 & 0.368 \\
\hline 11 & 1 & 111 & 18 & 0.008 & 0.094 & 16.225 & 0.437 \\
\hline । I & 1 & I I I & 19 & -0.084 & -0.026 & 16.795 & 0.468 \\
\hline I I & 1 & ID I & 20 & -0.067 & -0.180 & 17.174 & 0.511 \\
\hline
\end{tabular}

Fuente: Elaboración propia con datos de CNBV e INEGI

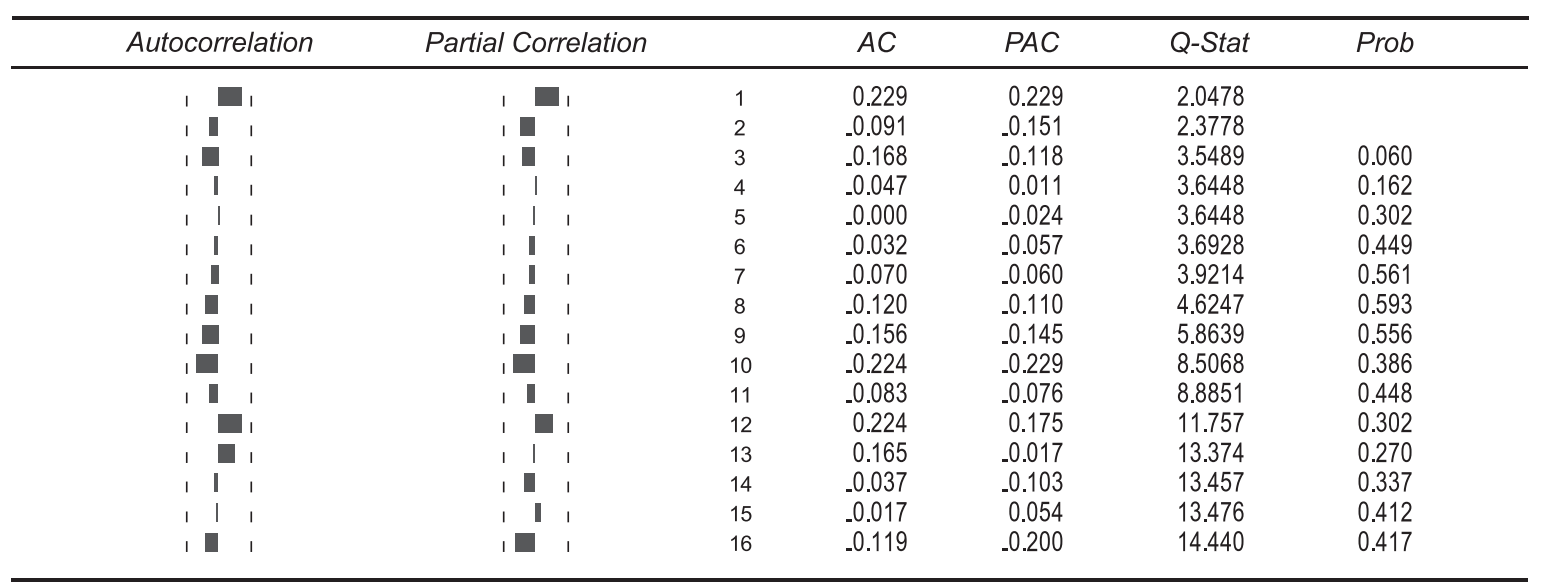

Fuente: Elaboración propia con datos de CNBV e INEGI 
Anexo 7

Gráficas de los residuos de los procesos $\mathrm{AR}(p)$ elegidos

R_IGAE

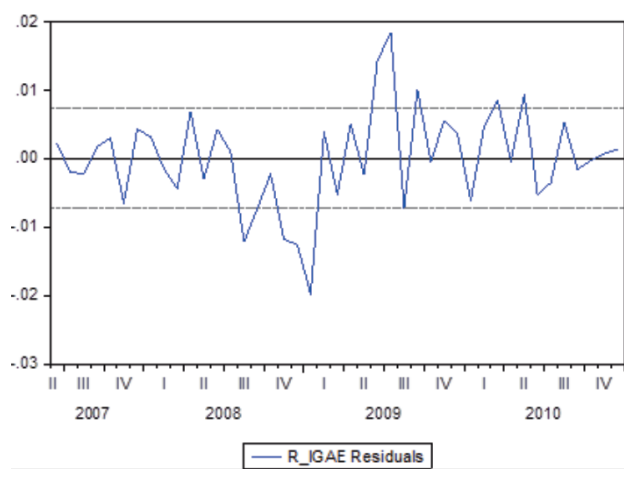

R_BBVA

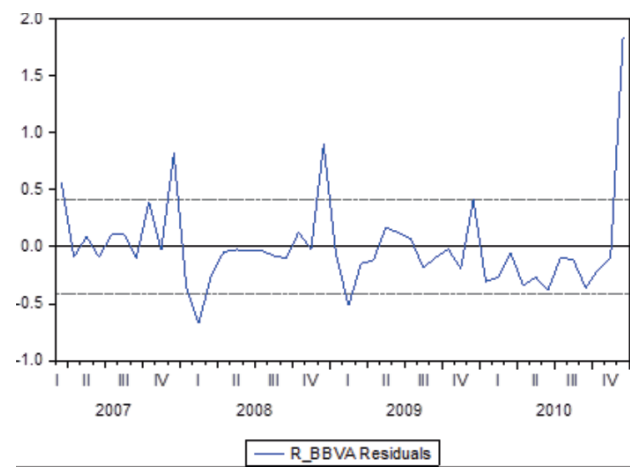

R_BANORTE

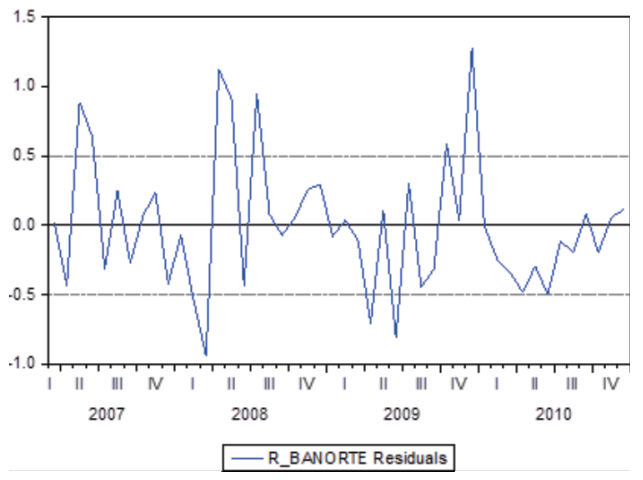

Fuente: Elaboración propia con datos de CNBV e INEGI 
Anexo 8

Estimación modelo MS1 2007 - 2010

\begin{tabular}{|c|c|c|c|c|}
\hline Variable & Coeficiente & Error Estándar & Estadístico z & Prob. \\
\hline \multicolumn{5}{|c|}{ Régimen 1} \\
\hline C & 0.56371 & 0.03127 & 18.02861 & 0.00000 \\
\hline $\mathrm{AR}(1)$ & -2.67425 & 0.25102 & -10.65364 & 0.00000 \\
\hline \multicolumn{5}{|c|}{ Régimen 2} \\
\hline C & -.0 .00362 & 0.02693 & -0.13427 & 0.89320 \\
\hline $\mathrm{AR}(1)$ & 0.01833 & 0.10528 & 0.17406 & 0.86180 \\
\hline \multicolumn{5}{|c|}{ Proceso no estacionario $A R$} \\
\hline
\end{tabular}

Fuente: Elaboración propia con datos de CNBV e INEGI 